\title{
SOBOLEV SPACES AND HYPERBOLIC FILLINGS
}

\author{
MARIO BONK AND EERO SAKSMAN
}

\begin{abstract}
Let $Z$ be an Ahlfors $Q$-regular compact metric measure space, where $Q>0$. For $p>1$ we introduce a new (fractional) Sobolev space $A^{p}(Z)$ consisting of functions whose extensions to the hyperbolic filling of $Z$ satisfy a weak-type gradient condition. If $Z$ supports a $Q$-Poincaré inequality with $Q>1$, then $A^{Q}(Z)$ coincides with the familiar (homogeneous) Hajłasz-Sobolev space.
\end{abstract}

\section{INTRODUCTION}

In this paper we consider Ahlfors $Q$-regular compact metric measure spaces $Z=(Z, d, \mu)$, where $Q>0$. We are interested in a certain Sobolev space on $Z$ and its relation to a function space that can be defined on a suitable hyperbolic filling of $Z$. Our results complement earlier work by Bourdon and Pajot $[\mathrm{BP}$, and by Connes, Semmes, Sullivan and Teleman [CST, Appendix]. In order to state our main theorems, we first have to discuss some basic concepts and set up some notation. More details can be found in the later sections.

The hyperbolic filling $X$ of $Z$ (see Section 3) is a simplicial graph $X=(V, E)$ with vertex set $V$ and edge set $E$. It carries a natural path metric obtained by identifying each edge $e$ in $X$ with a copy of the unit interval. Equipped with this metric, $X$ is Gromov hyperbolic and its boundary at infinity $\partial_{\infty} X$ can be identified with $Z$. The construction of $X$ depends on some choices, but $X$ is uniquely determined up to quasi-isometry.

In our construction, the vertex set $V$ is given by a collection of metric balls $B$ in $Z$. Then for an integrable function $f \in L^{1}(Z)$ (with $\mu$ being the underlying measure on $Z$ ) one can define the Poisson extension $u=P f: V \rightarrow \mathbb{R}$ by setting

$$
u(B)=\frac{1}{\mu(B)} \int_{B} f d \mu
$$

Date: March 28, 2015.

M.B. was supported by NSF grants DMS-0456940, DMS-0652915, DMS-1058283, DMS-1058772, and DMS-1162471.

E.S. was supported by the Finnish CoE in Analysis and Dynamics Research, and by the Academy of Finland, projects 113826 and 118765. 
for $B \in V$. For each edge $e \in E$ we choose one of the two vertices incident with $e$ as the initial point $e_{-}$and the other vertex as the terminal point $e_{+}$of $e$. If $u: V \rightarrow \mathbb{R}$ is a function on $V$, we can then define the gradient $d u: E \rightarrow \mathbb{R}$ of $u$ as

$$
d u(e)=u\left(e_{+}\right)-u\left(e_{-}\right)
$$

for $e \in E$. Note that both operators $f \mapsto P f$ and $u \mapsto d u$ are linear.

If $M$ is a countable set and $p \geq 1$, then we denote by $\ell^{p, \infty}(M)$ the weak-type $\ell^{p}$-space consisting of all functions $s: M \rightarrow \mathbb{R}$ such that there exists a constant $C \geq 0$ with

$$
\#\{m \in M:|s(m)|>\lambda\} \leq\left(\frac{C}{\lambda}\right)^{p}
$$

for all $\lambda>0$. For $p>1$ one can find a norm $\|s\|_{\ell^{p, \infty}}$ for such functions $s$ that is comparable to the infimum over all constants $C$ in the previous inequality (see Section 2).

Given these definitions one can define a Sobolev space as follows.

Definition 1.1. Let $p>1$. Then the Sobolev space $\dot{A}^{p}(Z)$ consists of all functions $f \in L^{1}(Z)$ such that the Poisson extension $u=P f$ satisfies $d u \in \ell^{p, \infty}(E)$. A semi-norm on this space is obtained by setting

$$
\|f\|_{\dot{A}^{p}}:=\|d(P f)\|_{\ell^{p, \infty}} .
$$

The semi-norm in (2) does not distinguish functions that differ by an additive constant; so $\dot{A}^{p}(Z)$ is a Sobolev space of homogeneous type. To promote the expression in (2) to a norm, we set $A^{p}(Z)=\dot{A}^{p}(Z) / \mathbb{R}$, where $\mathbb{R}$ stands for the space of (almost everywhere) constant functions on $Z$. Strictly speaking, the elements in $A^{p}(Z)$ are equivalence classes in $L^{1}(Z)$ modulo constant functions, but we prefer to represent an element in $A^{p}(Z)$ as an integrable function $f$ in $L^{1}(Z)$, defined up to an additive constant and up to changing the function on a set of measure zero. We set $\|f\|_{A^{p}}:=\|d(P f)\|_{\ell^{p, \infty}}$, which is well-defined on $A^{p}(Z)$.

Proposition 1.2. Let $p>1$. Then the map $f \in A^{p}(Z) \mapsto\|f\|_{A^{p}}$ defines a norm on $A^{p}(Z)$. With this norm, $A^{p}(Z)$ is a Banach space that is isomorphic to a closed subspace of $\ell^{p, \infty}(E)$.

By definition the space $A^{p}(Z)$ depends a priori on the choice of the hyperbolic filling $X=(V, E)$ of $Z$, but we will see that $A^{p}(Z)$ is independent of the choice of $X$ (see Corollary 4.6 and Remark 4.7).

One can find an isomorphism of the Sobolev space $A^{p}(Z)$ with a space that can be constructed from functions on the hyperbolic filling $X=(V, E)$. For this we fix $p>1$ and define an equivalence relation 
on real-valued functions on $V$ as follows: if $u, u^{\prime}: V \rightarrow \mathbb{R}$, we write $u \sim u^{\prime}$ if there exists a constant $c \in \mathbb{R}$ such that $u-u^{\prime}-c \in \ell^{p, \infty}(V)$. It is clear that $\sim$ is an equivalence relation and we denote by $[u]$ the corresponding equivalence class of a function $u: V \rightarrow \mathbb{R}$. We now define $\mathrm{Wk}^{1, p}(X)$ as the real vector space of all equivalence classes $[u]$ of functions $u: V \rightarrow \mathbb{R}$ with $d u \in \ell^{p, \infty}(E)$. So

$$
\mathrm{Wk}^{1, p}(X)=\left\{u: V \rightarrow \mathbb{R}: d u \in \ell^{p, \infty}(E)\right\} /\left(\mathbb{R}+\ell^{p, \infty}(V)\right),
$$

where $\mathbb{R}$ represents the space of constant functions on $V$. Note that if $u \in \mathbb{R}+\ell^{p, \infty}(V)$, then $d u \in \ell^{p, \infty}(E)$ (see Section 3). The space $\mathrm{Wk}^{1, p}(X)$ carries a natural semi-norm given by

$$
\|u\|_{\mathrm{Wk}^{1, p}}:=\inf \left\{\left\|d u^{\prime}\right\|_{\ell^{p, \infty}}: u^{\prime} \in[u]\right\} .
$$

Note that by definition $\|u\|_{\mathrm{Wk}^{1, p}}$ only depends on $[u]$ and not on the chosen representative $u$ in $[u]$. So $\|u\|_{\mathrm{Wk}^{1, p}}$ really defines a semi-norm on $\mathrm{Wk}^{1, p}(X)$, but for simplicity we suppress the distinction between $u$ and $[u]$ in our notation for the semi-norm.

It is easy to see that under some mild assumptions the space $\mathrm{Wk}^{1, p}(X)$ is invariant under quasi-isometries of $X$ (see Proposition 3.1).

If $p>1$, and $u: V \rightarrow \mathbb{R}$ is a function with $d u \in \ell^{p, \infty}(E)$, then one can show that it has a well-defined trace $\mathbb{R} u \in L^{1}(Z)$ on $Z=\partial_{\infty} X$ (see Lemma 4.1).

We can now state the main result of this paper.

Theorem 1.3. Let $p>1$, and $Z$ be an Ahlfors $Q$-regular space, where $Q>0$. Then the expression (41) defines a norm on $\mathrm{Wk}^{1, p}(X)$. Equipped with this norm, $\mathrm{Wk}^{1, p}(X)$ is a Banach space.

Moreover, the map $[u] \mapsto \mathbb{R} u$ is an isomorphism between the Banach spaces $\mathrm{Wk}^{1, p}(X)$ and $A^{p}(Z)$ with the inverse obtained from the Poisson extension by $f \mapsto[P f]$.

For general Ahlfors regular spaces the relation of our space $A^{p}(Z)$ to other known Sobolev-type spaces it not clear. It is easy to see that the (homogeneous) fractional Hajłasz-Sobolev $\dot{M}^{\alpha, p}(Z)$ is always contained in $\dot{A}^{p}(Z)$ for $\alpha=Q / p$ (see Section 5 ). If $Z$ satisfies a Poincaré inequality, then our space can be identified with the Hajłasz-Sobolev space for the endpoint case $p=Q$.

Theorem 1.4. Let $Z$ be an Ahlfors $Q$-regular compact metric measure space that supports a $Q$-Poincaré inequality, where $Q>1$. Then we have $\dot{A}^{Q}(Z)=\dot{M}^{1, Q}(Z)$, with comparability of semi-norms.

One can also show that under the assumptions of this theorem the space $\dot{A}^{p}(Z)$ consists only of (almost everywhere) constant functions for $1<p<Q$ (see Proposition [5.5). 
A reader with an orientation towards more classical analysis may wonder about analogs of our results in a standard Euclidean setting. We have included a discussion on this in Section 6. Here we will relate integrability properties of a function $f$ in $\mathbb{R}^{n}$ with weak-type conditions for the classical Poisson extension $u$ of $f$ defined on upper half-space $\mathbb{R}_{+}^{n+1}$ (see Proposition 6.1). This can be used to characterize functions $f$ with a distributional gradient in $L^{n}\left(\mathbb{R}^{n}\right)$ in terms of a weak-type condition of the hyperbolic gradient $\nabla_{h} u$; see Corollary 6.4 which corresponds to Theorem 1.4 .

The definition (3) of our space $\mathrm{Wk}^{1, p}(X)$ is similar to a definition in $\left[\mathrm{BP}\right.$, where the requirement is in terms of an $\ell^{p}$-condition instead of a weak-type condition. The space obtained in this way admits an identification with the $\ell^{p}$-cohomology of the hyperbolic filling $X$ in degree 1 and can also be identified with a Besov space $B_{p}(Z)$ on the boundary $Z=\partial_{\infty} X$. In $[\mathrm{BP}]$ it was also shown that in the setting as in Theorem 1.4, the space $B_{p}(Z)$ is interesting only for $p>Q$, because it is trivial and consists of constant functions in the endpoint case $p=Q$. Theorem 1.4 suggests that one should modify the definition of $\ell^{p}$-cohomology if one wants to obtain interesting function spaces for critical exponents. Namely, one can set up a cohomology theory for (infinite) simplicial complexes (satisfying additional natural geometric conditions), where the requirement is that cochains belong to $\ell^{p, \infty}$ instead of $\ell^{p}$. Our notation for the space $\mathrm{Wk}^{1, p}(X)$ is suggested by the fact that it represents weak-type $\ell^{p}$-cohomology in degree 1. Many important features of $\ell^{p}$-cohomology such as quasi-isometric invariance properties remain valid in this context of weak-type $\ell^{p}$-cohomology. This is an interesting direction to pursue, but we will not do this in this paper.

Our paper is organized as follows. In Section 2 we review some facts about sequence spaces and weak-type conditions. We consider the space $\mathrm{Wk}^{1, p}(X)$ on general simplicial graphs $X$ in Section 3 , where we prove a quasi-isometric invariance property. In Section 4 we specialize to Ahlfors regular spaces $Z$ and their hyperbolic fillings. There we will prove Proposition 1.2 and Theorem [1.3. In Section 5 we discuss Sobolev spaces and their relation to our space $\dot{A}^{p}(Z)$. In particular, we give a proof of Theorem 1.4. As was already mentioned, Section 6 is devoted to results about the classical Poisson extension in $\mathbb{R}^{n}$.

Acknowledgement. The authors are indebted to Marc Bourdon, Bruce Kleiner, Pierre Pansu, and Tomas Soto for many interesting discussions relating to the topic of this paper. They also thank Jeff Lindquist for a careful reading of a draft of the paper. 


\section{SEquenCE SPACES}

In the following, we will extensively use the notation $A \lesssim B, A \gtrsim B$, and $A \simeq B$ for quantities $A$ and $B$ to indicate the existence of an implicit constant $C \geq 1$ depending on some inessential parameters such $A \leq C B, A \geq B / C$ and $A / C \leq B \leq C A$, respectively. Of course, it depends on the context which parameters can be safely ignored. We will mostly leave it to the judicious readers to make their own judgements about this.

For $p \in[1, \infty)$ we denote by $\ell^{p}$ the space of all real-valued sequences $s=\left\{x_{n}\right\} \in \mathbb{R}^{\mathbb{N}}$ such that

$$
\|s\|_{\ell^{p}}:=\left(\sum_{n=1}^{\infty}\left|x_{n}\right|^{p}\right)^{1 / p}<\infty .
$$

Note that $\|s\|_{\ell^{q}} \leq\|s\|_{\ell^{p}}$ and so $\ell^{p} \subset \ell^{q}$ for $1 \leq p \leq q$.

We denote by $\ell^{p, \infty}$ the space of all sequences $s=\left\{x_{n}\right\} \in \mathbb{R}^{\mathbb{N}}$ for which there exists a constant $C \geq 0$ with

$$
\#\left\{n \in \mathbb{N}:\left|x_{n}\right|>\lambda\right\} \leq\left(\frac{C}{\lambda}\right)^{p}
$$

for all $\lambda>0$. Here $\# M \in \mathbb{N}_{0} \cup\{\infty\}$ denotes the cardinality of a set $M$. Note that $\ell^{p} \subset \ell^{p, \infty}$.

For a given sequence $s=\left\{x_{n}\right\} \in \mathbb{R}^{\mathbb{N}}$ we denote the infimum of all constants $C$ for which (5) is valid as $\|s\|_{\ell^{p, \infty}}^{*}$. We then have the homogeneity property $\|a s\|_{\ell^{p, \infty}}^{*}=|a| \cdot\|s\|_{\ell^{p, \infty}}^{*}$ for $a \in \mathbb{R}$, but we do not have subadditivity. So $\|s\|_{\ell^{p, \infty}}^{*}$ does not define a norm on $\ell^{p, \infty}$. For $p>1$ we consider the expression

$$
\|s\|_{\ell^{p, \infty}}:=\sup \left\{n^{-1+1 / p} \sum_{j \in E}\left|x_{j}\right|: n \in \mathbb{N}, E \subset \mathbb{N}, \# E=n\right\}
$$

The quantities $\|s\|_{\ell^{p, \infty}}^{*}$ and $\|s\|_{\ell^{p, \infty}}$ are comparable (see Lemma 2.1 (i) below) and it is easy to verify that $\|s\|_{\ell^{p, \infty}}$ defines a norm on $\ell^{p, \infty}$. We will equip $\ell^{p, \infty}$ with this norm in the following, but we will freely switch between $\|s\|_{\ell^{p}, \infty}$ and the comparable expression $\|s\|_{\ell^{p, \infty}}^{*}$ whenever convenient. The space $\ell^{p, \infty}$ is a Banach space, and one can show that it is non-reflexive (see [Gr, Section 1.4] for general background on weak $L^{p}$-spaces).

We will also consider sequences indexed by more general countable index sets $M$, or, more precisely, functions $s: M \rightarrow \mathbb{R}$. For clarity we will then denote the corresponding spaces by $\ell^{p}(M)$ and $\ell^{p, \infty}(M)$, but will suppress $M$ in the notation for norms.

We need several simple observations. 
Lemma 2.1. (i) For each $p>1$ there exists a constant $C \geq 1$ such that

$$
\|s\|_{\ell^{p, \infty}}^{*} \leq\|s\|_{\ell^{p, \infty}} \leq C\|s\|_{\ell^{p, \infty}}^{*}
$$

for all $s \in \mathbb{R}^{\mathbb{N}}$.

(ii) Let $p>1$ and $r \in[1, p)$. Then there exists a constant $C^{\prime}>0$ such for every sequence $s=\left\{x_{1}, \ldots, x_{m}, 0,0, \ldots\right\} \in \mathbb{R}^{\mathbb{N}}$ with $m \in \mathbb{N}$ we have

$$
\|s\|_{\ell^{p}} \leq C^{\prime}(1+\log m)^{1 / p}\|s\|_{\ell^{p, \infty}} \quad \text { and } \quad\|s\|_{\ell^{r}} \leq C^{\prime} m^{1 / r-1 / p}\|s\|_{\ell^{p, \infty}} .
$$

Proof. (i) Let $s=\left\{x_{n}\right\} \in \mathbb{R}^{\mathbb{N}}$ be arbitrary.

To show the first inequality, we may assume $\|s\|_{\ell^{p, \infty}}=1$. If $\lambda>0$ and $E \subset \mathbb{N}$ is a finite set such that $\left|x_{j}\right|>\lambda$ for $j \in E$, we then have

$$
\lambda(\# E) \leq \sum_{j \in E}\left|x_{j}\right| \leq(\# E)^{1-1 / p} .
$$

It follows that $\# E \leq 1 / \lambda^{p}$. This implies $\|s\|_{\ell p, \infty}^{*} \leq 1$ as desired.

To establish the second inequality, we may assume that $\|s\|_{\ell p, \infty}^{*}=1$. Let $E \subset \mathbb{N}$ be an arbitrary finite non-empty set and set $m=\# E$. Then for each $k \in \mathbb{Z}$ the number of elements $j \in E$ with $\left|x_{j}\right| \in\left(2^{-k}, 2^{1-k}\right]$ is bounded above by $\min \left\{m, 2^{p k}\right\}$. Hence

$$
\sum_{j \in E}\left|x_{j}\right| \leq \sum_{k=0}^{\infty} 2^{1-k} \min \left\{m, 2^{p k}\right\} \leq C m^{1-1 / p} .
$$

Here $C \geq 1$ can be chosen only depending on $p$. It follows that $\|s\|_{\ell^{p, \infty}} \leq C$ as desired.

(ii) Let $s \in \mathbb{R}^{\mathbb{N}}$ be as in the statement. We may assume $\|s\|_{\ell^{p, \infty}}=1$ which implies $\|s\|_{\ell^{p}, \infty}^{*} \leq 1$ by (i). Then, similarly as in the proof of (i), for each $k \in \mathbb{Z}$ the number of elements $x_{j}$ in the sequence $s$ with $\left|x_{j}\right| \in\left(2^{-k}, 2^{1-k}\right]$ is bounded above by $\min \left\{m, 2^{p k}\right\}$. Hence

$$
\|s\|_{\ell^{p}}^{p}=\sum_{j=1}^{m}\left|x_{j}\right|^{p} \lesssim \sum_{k=0}^{\infty} 2^{-p k} \min \left\{m, 2^{p k}\right\} \lesssim 1+\log m .
$$

The other estimate follows from a similar computation.

Lemma 2.2. Let $p>1$ and $E \subset \mathbb{N} \times \mathbb{N}$. For $k, n \in \mathbb{N}$ define

$$
E_{n}=\{k \in \mathbb{N}:(n, k) \in E\} \text { and } E^{k}=\{n \in \mathbb{N}:(n, k) \in E\},
$$

and suppose that for some $N \in \mathbb{N}$ we have $\# E_{n} \leq N$ and $\# E^{k} \leq N$ for all $k, n \in \mathbb{N}$. Then there exists a constant $C=C(p, N)>0$ with the following property: 
If $s=\left\{x_{n}\right\}$ and $t=\left\{y_{n}\right\}$ are sequences in $\mathbb{R}^{\mathbb{N}}$ such that

$$
\left|x_{n}\right| \leq \sum_{k \in E_{n}}\left|y_{k}\right|
$$

for all $n \in \mathbb{N}$, then $\|s\|_{\ell^{p, \infty}} \leq C(p, N)\|t\|_{\ell^{p, \infty}}$.

Proof. We may assume $\|t\|_{\ell^{p, \infty}}^{*}=1$. Let $\lambda>0$ and suppose that $\left|x_{n}\right|>$ $\lambda$. Then there exists $k \in E_{n}$ such that $\left|y_{k}\right|>\lambda / N$. Each such $k$ lies in at most $\# E^{k} \leq N$ sets $E_{n}$. Hence

$$
\#\left\{n \in \mathbb{N}:\left|x_{n}\right|>\lambda\right\} \leq N \#\left\{k \in \mathbb{N}:\left|y_{k}\right|>\lambda / N\right\} \leq N^{p+1} / \lambda^{p} .
$$

The claim follows.

In the next lemma it is convenient to view a sequence $s \in \mathbb{R}^{\mathbb{N}}$ as a function $s: \mathbb{N} \rightarrow \mathbb{R}$.

Lemma 2.3. Let $p \geq 1$ and suppose that $s$ and $s_{k}$ for $k \in \mathbb{N}$ are sequences in $\mathbb{R}^{\mathbb{N}}$ such that for each $n \in \mathbb{N}$ we have $s_{k}(n) \rightarrow s(n)$ as $k \rightarrow \infty$. Then

$$
\|s\|_{\ell^{p, \infty}}^{*} \leq \liminf _{k \rightarrow \infty}\left\|s_{k}\right\|_{\ell^{p, \infty}}^{*}
$$

In particular, if $\left\|s_{k}\right\|_{\ell^{p, \infty}}$ is uniformly bounded for $k \in \mathbb{N}$, then $s \in$ $\ell^{p, \infty}$.

Proof. If $C_{0}:=\liminf _{k \rightarrow \infty}\left\|s_{k}\right\|_{\ell^{p}, \infty}^{*}=\infty$, there is nothing to prove. So suppose that $C_{0}<\infty$. Pick $C>C_{0}$. By passing to a subsequence if necessary, we may assume that $\left\|s_{k}\right\|_{\ell^{p}, \infty}^{*}<C$ for all $k \in \mathbb{N}$. Let $\lambda>0$ be arbitrary. If $|s(n)|>\lambda$ for some $n \in \mathbb{N}$, then $\left|s_{k}(n)\right|>\lambda$ for all sufficiently large $k$. Hence

$$
\#\{n \in \mathbb{N}:|s(n)|>\lambda\} \leq \liminf _{k \rightarrow \infty} \#\left\{n \in \mathbb{N}:\left|s_{k}(n)\right|>\lambda\right\} \leq(C / \lambda)^{p},
$$

and so $\|s\|_{\ell^{p, \infty}}^{*} \leq C$. If we let $C \rightarrow C_{0}$, the claim follows.

\section{FunCTION SPACES ON SIMPLICIAL GRAPHS}

Before we turn to hyperbolic fillings of Ahlfors regular spaces, we will discuss some facts for general simplicial graphs $X=(V, E)$. We assume that $X$ is connected and carries a path metric obtained by identifying each edge $e \in E$ with a copy of the unit interval [0,1]. We will also assume that each pair of vertices in $V$ is joined by at most one edge in $E$ and that the degree of each vertex $v \in V$, i.e., the number of edges incident with $v$, is uniformly bounded from above. This implies that if $R>0$, then the number of edges and vertices contained in a ball $B \subset X$ of radius $R$ is uniformly bounded above only depending on $R$.

As already mentioned in the introduction, it is convenient choose one of the vertices incident with $e \in E$ as the initial point $e_{-}$and the other 
as the terminal point $e_{+}$of $e$. For a function $u: V \rightarrow \mathbb{R}$, we define the gradient $d u: E \rightarrow \mathbb{R}$ of $u$ as in (1). Then for each $e \in E$ we have

$$
|d u(e)|=\left|u\left(e_{+}\right)-u\left(e_{-}\right)\right| \leq\left|u\left(e_{+}\right)\right|+\left|u\left(e_{-}\right)\right| .
$$

Here each vertex $v \in V$ appears as an endpoint $e_{+}$or $e_{-}$only for a uniformly bounded number of edges $e$. Hence Lemma 2.2 implies that if $p>1$, then

$$
\|d u\|_{\ell^{p, \infty}} \leq C\|u\|_{\ell^{p, \infty}}
$$

with a constant $C>0$ independent of $u$. In other words, the map $u \mapsto$ $d u$ is a bounded linear operator $d: \ell^{p, \infty}(V) \rightarrow \ell^{p, \infty}(E)$. In particular, $\mathbb{R}+\ell^{p, \infty}(V)$ is is a subspace of $\left\{u: V \rightarrow \mathbb{R}: d u \in \ell^{p, \infty}(E)\right\}$ and one can define $\mathrm{Wk}^{1, p}(X)$ as in (3) for each $p>1$. The space $\mathrm{Wk}^{1, p}(X)$ carries the semi-norm as defined in (4).

Two metric spaces $\left(X, d_{X}\right)$ and $\left(Y, d_{Y}\right)$ are called quasi-isometric if there exists a map $\varphi: X \rightarrow Y$ such that for some constants $\lambda \geq 1$ and $K \geq 0$ we have

$$
\frac{1}{\lambda} d_{X}\left(x, x^{\prime}\right)-K \leq d_{Y}\left(\varphi(x), \varphi\left(x^{\prime}\right)\right) \leq \lambda d_{X}\left(x, x^{\prime}\right)+K
$$

for all $x, x^{\prime} \in X$, and

$$
\sup _{y \in Y} \inf _{x \in X} d_{Y}(y, \varphi(x)) \leq K .
$$

A map $\varphi: X \rightarrow Y$ as in this definition is called a quasi-isometry.

Proposition 3.1. Suppose $X=(V, E)$ and $X^{\prime}=\left(V^{\prime}, E^{\prime}\right)$ are simplicial metric graphs that are connected and have uniformly bounded vertex degree. If $X$ and $X^{\prime}$ are quasi-isometric, then for each $p>1$ the spaces $\mathrm{Wk}^{1, p}(X)$ and $\mathrm{Wk}^{1, p}\left(X^{\prime}\right)$ are isomorphic.

Proof. We will show that there exists a linear bijection between the function spaces that gives comparability of the semi-norms.

Our assumptions imply that there exist quasi-isometries $\psi: V^{\prime} \rightarrow V$ and $\varphi: V \rightarrow V^{\prime}$ that are coarse inverses of each other; more precisely, $\psi \circ \varphi$ and $\varphi \circ \psi$ are in bounded distance to the identity map on $V$ and $V^{\prime}$, respectively.

Then any two preimages of a vertex $v \in V$ under $\psi$ have uniformly bounded distance. Since the vertex degree of $X$ is uniformly bounded, we conclude that there exists a constant $N \in \mathbb{N}$ such that $\# \psi^{-1}(v) \leq N$ for all $v \in V$. This implies that if $u \in \mathbb{R}+\ell^{p, \infty}(V)$, then $u \circ \psi \in$ $\mathbb{R}+\ell^{p, \infty}\left(V^{\prime}\right)$.

Suppose $u: V \rightarrow \mathbb{R}$ and $d u \in \ell^{\infty}(E)$. If $e^{\prime} \in E^{\prime}$, then the vertices $e_{+}^{\prime}$ and $e_{-}^{\prime}$ are adjacent in $V^{\prime}$. Then they have distance 1 in $X^{\prime}$, and so their images $\psi\left(e_{+}^{\prime}\right)$ and $\psi\left(e_{-}^{\prime}\right)$ have uniformly bounded distance in 
$V$. We join these images by a geodesic segment, and estimate their function value difference by the triangle inequality. It then follows that for a radius $R_{0}>0$ independent of $e^{\prime}$ we have

$$
\left|d(u \circ \psi)\left(e^{\prime}\right)\right|=\mid\left(u\left(\psi\left(e_{+}^{\prime}\right)\right)-u\left(\psi\left(e_{-}^{\prime}\right)\right)\left|\leq \sum_{e \subset B\left(\psi\left(e_{+}^{\prime}\right), R_{0}\right)}\right| d u(e) \mid,\right.
$$

where $B(a, r) \subset X$ denotes the open ball of radius $r>0$ centered at $a \in X$.

The number of the edges $e$ contributing to the last sum is uniformly bounded independent of $e^{\prime}$; moreover, if a given edge $e \in E$ contributes, then $e_{+}$and $\psi\left(e_{+}^{\prime}\right)$ have uniformly bounded distance. If we apply the coarse inverse $\varphi$ of $\psi$ here, we see that $\varphi\left(e_{+}\right)$and $e_{+}^{\prime}$ have uniformly bounded distance; so for given $e \in E$ there is uniformly bounded number of edges $e^{\prime} \in E^{\prime}$ so that $e$ contributes to the sum (8). These considerations show that we can apply Lemma 2.2 and we conclude that

$$
\|d(u \circ \psi)\|_{\ell^{p, \infty}} \leq C\|d u\|_{\ell^{p, \infty}} .
$$

with a constant $C \geq 0$ independent of $u$.

By what we have seen, the linear map

$$
[u] \in \mathrm{Wk}^{1, p}(X) \mapsto[u \circ \psi] \in \mathrm{Wk}^{1, p}\left(X^{\prime}\right)
$$

is well-defined, and we get a uniform semi-norm bound

$$
\|u \circ \psi\|_{\mathrm{Wk}^{1, p}} \leq C\|u\|_{\mathrm{Wk}^{1, p}} .
$$

Of course, the roles of $\varphi$ and $\psi$ are completely symmetric, and we can apply the previous considerations to $\varphi$. So we get a well-defined linear map

$$
\left[u^{\prime}\right] \in \mathrm{Wk}^{1, p}\left(X^{\prime}\right) \mapsto\left[u^{\prime} \circ \varphi\right] \in \mathrm{Wk}^{1, p}(X)
$$

with a corresponding semi-norm bound. To finish the proof, it is enough to show that the maps $[u] \mapsto[u \circ \psi]$ and $\left[u^{\prime}\right] \mapsto\left[u^{\prime} \circ \psi\right]$ are inverse to each other, or equivalently that $[u]=[u \circ \psi \circ \varphi]$ and $\left[u^{\prime}\right]=\left[u^{\prime} \circ \varphi \circ \psi\right]$. By symmetry, it is enough to show that $[u]=[u \circ \psi \circ \varphi]$ for $[u] \in \mathrm{Wk}^{1, p}(X)$.

So let $u: V \rightarrow \mathbb{R}$ with $d u \in \ell^{p, \infty}(E)$ be arbitrary. If $v \in V$, then $(\psi \circ \varphi)(v) \in B\left(v, R_{1}\right)$, where $R_{1}$ is independent of $v$. Hence

$$
|u(v)-(u \circ \psi \circ \varphi)(v)| \leq \sum_{e \subset B\left(v, R_{1}\right)}|d u(e)| .
$$

Again there is only a uniformly bounded number of edges $e$ contributing to this sum, and a given edge $e$ can only appear for a uniformly bounded number of vertices $v \in V$. So by Lemma 2.2 we have

$$
\|u-u \circ \psi \circ \varphi\|_{\ell^{p, \infty}} \lesssim\|d u\|_{\ell^{p, \infty}}<\infty .
$$


In particular, $u-u \circ \psi \circ \varphi \in \ell^{p, \infty}(V)$, and so $[u]=[u \circ \psi \circ \varphi]$ as desired.

\section{Ahlfors Regular SPACES AND HYPERBoliC Fillings}

In this and the following sections $Z=(Z, d, \mu)$ is an Ahlfors $Q$ regular compact metric measure space, where $Q>0$. Here $d$ is a metric and $\mu$ a Borel measure on $Z$. We will assume that the diameter $\operatorname{diam}(Z)$ of $Z$ is equal to 1 . This can always be achieved by a possible rescaling of the metric. We use both $\mu(E)$ and $|E|$ to denote the measure of a (Borel) set $E \subset Z$. Ahlfors $Q$-regularity then means that $|B| \simeq r^{Q}$ whenever $B$ is a ball of radius $r \leq 1$ in $Z$.

If $a \in Z$ and $r>0$, we denote by $B(a, r)$ the open ball in $Z$ of radius $r$ centered at $a$. If $\Lambda \geq 1$ and $B=B(a, r)$ is a ball, we set $\Lambda B=B(a, \Lambda r)$. For $p \geq 1$ we denote by $L^{p}(Z)$ the space of all measurable functions $f: Z \rightarrow \mathbb{R}$ such that

$$
\|f\|_{L^{p}}:=\left(\int_{Z}|f|^{p} d \mu\right)^{1 / p}<\infty .
$$

If $f \in L^{1}(Z)$ and $B$ is a ball in $Z$, we define

$$
f_{B}=\frac{1}{|B|} \int_{B} f d \mu .
$$

We will now review the construction of the hyperbolic filling of $Z$. We will mostly follow [BP, Section 2.1] (see also [BS, Chapter 6]). Note that we can apply the considerations in $[\mathrm{BP}$, because as an Ahlfors regular space, $Z$ is doubling and uniformly perfect (see [He, Sections 10.13 and 11.1] for this terminology).

For each $n \in \mathbb{N}$ we choose a maximal $2^{-n}$-separated set $Z_{n}$ of points in $Z$. Then the balls $B\left(z, 2^{-n}\right), z \in Z_{n}$, form a cover of $Z$. The hyperbolic filling is a graph that essentially records the incidence relations of the balls in these covers. In order to get good properties it is necessary to enlarge the radii of these balls by a factor $\Lambda>1$ (this was overlooked in $[\mathrm{BP}$, Section 2.1], and causes problems in the proof of [BP, Lemme $2.2]$, but the argument is valid if one uses enlarged balls).

Accordingly, for $n \in \mathbb{N}$ we let $V_{n}$ be the collection of all balls $B=$ $B\left(z, 2^{1-n}\right)$ with $z \in Z_{n}$, where we refer to $n$ as the level of the ball $B$. Here we add $B=Z$ as a ball on level 0 and set $V_{0}=\{Z\}$. The vertex set $V$ of our graph is the (disjoint) union of the sets $V_{n}, n \in \mathbb{N}_{0}$. We join two distinct vertices as represented by balls $B$ and $B^{\prime}$ by an edge $e$ if their levels differ by at most 1 and if $B \cap B^{\prime} \neq \emptyset$. We write $B^{\prime} \sim B$ in this case and denote by $E$ the set of these edges $e$. The simplicial graph $X=(V, E)$ carries a natural path metric obtained by identifying 
each edge $e$ in $X$ with a unit interval. Then the graph $X$ equipped with this metric is Gromov hyperbolic and the boundary at infinity $\partial_{\infty} X$ of $X$ can be identified with $Z$. The graph $X$ is also connected and the degree of each vertex is uniformly bounded from above. In particular, we can apply the considerations in Section 3 to $X$.

The construction of $X=(V, E)$ depends on choices, namely on the sets $Z_{n}$. One can also choose a scale sequence $\Lambda^{-n}$ with a different parameter $\Lambda>1$, instead of the sequence $2^{-n}$ (as in [BS] and [BP]). These constructions lead to hyperbolic fillings that are quasi-isometric to our filling $X$ of $Z$ (this can easily be proved directly or one can invoke a general fact such as $[\mathrm{BP}$, Théorème 2.3]). By Proposition 3.1 this quasi-isometric ambiguity is irrelevant for the function spaces $\mathrm{Wk}^{1, p}(X)$ we are interested in.

For $n \in \mathbb{N}$ the set $V_{n}$ is the sphere of radius $n$ centered at $B_{0}=Z \in V$ in the subset $V$ of the hyperbolic filling $X$. Since $Z$ is Ahlfors $Q$-regular, we have $\# V_{n} \simeq 2^{Q n}$.

We make the conventions underlying the definition in (1). It is useful to also use variants of the gradient $d u$; namely, if $u: V \rightarrow \mathbb{R}$ is arbitrary, we define $\tilde{d} u: V \rightarrow \mathbb{R}$ by

$$
\tilde{d} u(B)=\sum_{B^{\prime} \in V, B^{\prime} \sim B}\left|u\left(B^{\prime}\right)-u(B)\right|
$$

for $B \in V$, and $\tilde{d}_{n} u: V_{n} \rightarrow \mathbb{R}$ as the restriction of $\tilde{d} u$ to $V_{n}$ for $n \in \mathbb{N}_{0}$. Note that in contrast to the gradient $d u$, the function $\tilde{d} u$ is defined on $V$ and not on $E$ and $u \mapsto \tilde{d} u$ is not a linear map.

If $p \geq 1$ and $u: V \rightarrow \mathbb{R}$ is arbitrary, then for each $B \in V$ we have

$$
\tilde{d} u(B) \leq \sum\left\{|d u(e)|: e \in E \text { and } B=e_{+} \text {or } B=e_{-}\right\} .
$$

By Lemma 2.2 this implies $\|\tilde{d} u\|_{\ell^{p, \infty}} \lesssim\|d u\|_{\ell^{p, \infty}}$. In the other direction, we have $|d u(e)| \leq \tilde{d} u\left(e_{-}\right)$for $e \in E$, which again by Lemma 2.2 implies that $\|d u\|_{\ell^{p, \infty}} \lesssim\|\tilde{d} u\|_{\ell^{p, \infty}}$. It follows that

$$
\|\tilde{d} u\|_{\ell^{p, \infty}} \simeq\|d u\|_{\ell^{p, \infty}}
$$

with an implicit multiplicative constant independent of $u: V \rightarrow \mathbb{R}$.

For each $n \in \mathbb{N}$ the balls $\frac{1}{2} B$ with $B \in V_{n}$ form an open cover of $Z$. Accordingly, we can choose a Lipschitz partition of unity on $Z$ given by finitely many non-negative Lipschitz functions $\left\{\psi_{B}\right\}_{B \in V_{n}}$ on $Z$ such that

$$
\begin{gathered}
\sum_{B \in V_{n}} \psi_{B}=1, \\
\operatorname{supp}\left(\psi_{B}\right):=\overline{\{u \in Z: \psi(u) \neq 0\}} \subset B,
\end{gathered}
$$


and

$$
\operatorname{Lip}\left(\psi_{B}\right):=\sup \left\{\frac{\left|\psi_{B}(u)-\psi_{B}(v)\right|}{d(u, v)}: u, v \in Z, u \neq v\right\} \lesssim 2^{n} .
$$

See [Se, p. 431, B.7.4 Lemma]) for the existence of such a partition of unity (the inclusion (10) which is stronger than in this reference can easily be obtained from a slight modification in the proof).

For the ball $B=Z$ on level 0 we choose $\psi_{B}=1$.

For $u: V \rightarrow \mathbb{R}$ we define a function $T_{n} u: Z \rightarrow \mathbb{R}$ as

$$
T_{n} u=\sum_{B \in V_{n}} u(B) \psi_{B} .
$$

Then for $n \in \mathbb{N}_{0}$ we have

$$
\begin{aligned}
\left|T_{n} u-T_{n+1} u\right| & \leq \sum_{B \in V_{n}} \sum_{B^{\prime} \in V_{n+1}}\left|u(B)-u\left(B^{\prime}\right)\right| \psi_{B} \psi_{B^{\prime}} \\
& \leq \sum_{B \in V_{n}} \tilde{d} u(B) \psi_{B} .
\end{aligned}
$$

Note that the last inequality follows from the fact that $\psi_{B} \psi_{B^{\prime}}=0$ for $B \in V_{n}$ and $B^{\prime} \in V_{n+1}$ unless $B \cap B^{\prime} \neq \emptyset$ and so $B \sim B^{\prime}$.

Now $\left\|\psi_{B}\right\|_{L^{1}} \lesssim 2^{-n Q}$ for $B \in V_{n}$, and so we obtain

$$
\left\|T_{n} u-T_{n+1} u\right\|_{L^{1}} \lesssim 2^{-n Q}\left\|\tilde{d}_{n} u\right\|_{\ell^{1}} .
$$

The following lemma provides an inverse operation to the Poisson extension.

Lemma 4.1. Let $p>1$ and $u: V \rightarrow \mathbb{R}$ with $d u \in \ell^{p, \infty}(E)$. Then

$$
\sum_{n=0}^{\infty}\left\|T_{n+1} u-T_{n} u\right\|_{L^{1}} \lesssim\|d u\|_{\ell^{p, \infty}} .
$$

In particular, the limit

$$
\mathbb{R} u:=\lim _{n \rightarrow \infty} T_{n} u .
$$

exists both in $L^{1}(Z)$ and pointwise almost everywhere in $Z$.

Proof. We have $\# V_{n} \lesssim 2^{n Q}$, and so Lemma 2.1 (ii) and (9) imply that

$$
\left\|\tilde{d}_{n} u\right\|_{\ell^{1}} \lesssim\left(2^{n Q}\right)^{1-1 / p}\|d u\|_{\ell^{p, \infty}} .
$$

Hence by (13),

$$
\sum_{n=0}^{\infty}\left\|T_{n+1} u-T_{n} u\right\|_{L^{1}} \lesssim \sum_{n=0}^{\infty} 2^{-n Q}\left\|\tilde{d}_{n} u\right\|_{\ell^{1}}
$$




$$
\lesssim \sum_{n=0}^{\infty} 2^{-n Q / p}\|d u\|_{\ell^{p, \infty}} \lesssim\|d u\|_{\ell^{p, \infty}} .
$$

The claim easily follows.

Lemma 4.2. Let $p>1$. If $f \in L^{1}(Z)$ and $d(P f) \in \ell^{p, \infty}(E)$, then $f=\mathbb{R}(P f)$. Moreover,

$$
\left\|f-\frac{1}{|Z|} \int_{Z} f d \mu\right\|_{L^{1}} \lesssim\|d(P f)\|_{\ell^{p, \infty}} .
$$

Proof. To prove the first part, let $\epsilon>0$ be arbitrary. We know that $T_{n}(P f) \rightarrow \mathbb{R}(P f)$ in $L^{1}(Z)$ as $n \rightarrow \infty$ by Lemma 4.1, and so $\left\|T_{n}(P f)-\mathbb{R}(P f)\right\|_{L^{1}}<\epsilon$ for large $n$. Since continuous functions are dense in $L^{1}(Z)$, we can find a continuous function $g$ on $Z$ with $\|g-f\|_{L^{1}}<\epsilon$. It is clear that $T_{n}(P g) \rightarrow g$ uniformly on $Z$ and hence also $\left\|T_{n}(P g)-g\right\|_{L^{1}}<\epsilon$ for large $n$.

Note that if $h \in L^{1}(Z)$, then

$$
\left\|T_{n}(P h)\right\|_{L^{1}} \lesssim \sum_{B \in V_{n}}\left\|\psi_{B}\right\|_{L^{1}}|P h(B)| \lesssim \sum_{B \in V_{n}}|B||P h(B)| \lesssim\|h\|_{L^{1}}
$$

If we apply this to $h=f-g$, then for some large $n \in \mathbb{N}$ we have

$$
\begin{gathered}
\|\mathbb{R}(P f)-f\|_{L^{1}} \leq\left\|\mathbb{R}(P f)-T_{n}(P f)\right\|_{L^{1}}+\left\|T_{n}(P(f-g))\right\|_{L^{1}}+ \\
\left\|T_{n}(P g)-g\right\|_{L^{1}}+\|g-f\|_{L^{1}} \lesssim \epsilon .
\end{gathered}
$$

Since $\epsilon>0$ was arbitrary, we conclude that $f=\mathbb{R}(P f)\left(\right.$ in $L^{1}(Z)$, i.e., the functions agree almost everywhere on $Z$ ).

For the second part we note that

$$
\frac{1}{|Z|} \int_{Z} f d \mu=T_{0}(P f)
$$

because $V_{0}=\{Z\}$. So if we use Lemma 4.1, then we obtain

$$
\begin{aligned}
\left\|f-\frac{1}{|Z|} \int_{Z} f d \mu\right\|_{L^{1}} & =\left\|f-T_{0}(P f)\right\|_{L^{1}} \\
& \leq \sum_{n=0}^{\infty}\left\|T_{n+1}(P f)-T_{n}(P f)\right\|_{L^{1}} \\
& \lesssim\|d(P f)\|_{\ell^{p, \infty}} .
\end{aligned}
$$

Proof of Proposition 1.2. Let $f \in L^{1}(Z)$. Since $X$ is connected, we have $d(P f)=0$ if and only if $P f$ is constant on $V$. This happens precisely if $f$ is constant (almost everywhere on $Z$ ). In particular, $\|f\|_{A^{p}}=\|d(P f)\|_{\ell^{p, \infty}}$ defines a norm on $A^{p}(Z)$, and the linear map $f \mapsto d(P f)$ gives an isometric embedding of $A^{p}(Z)$ into $\ell^{p, \infty}(E)$. To 
finish the proof, it is enough to show that every Cauchy sequence $\left\{f_{n}\right\}$ in $A^{p}(Z)$ converges to an element in $A^{p}(Z)$ (then the isometric image of $A^{p}(Z)$ in $\ell^{p, \infty}(E)$ is closed). Since $f_{n}$ is only well-defined up to a constant, we may assume that $\int_{Z} f_{n} d \mu=0$. Then Lemma 4.2 shows that

$$
\left\|f_{n}-f_{k}\right\|_{L^{1}} \lesssim\left\|d\left(P\left(f_{n}-f_{k}\right)\right)\right\|_{\ell^{p, \infty}}
$$

for $k, n \in \mathbb{N}$. Hence $\left\{f_{n}\right\}$ is a Cauchy sequence in $L^{1}(Z)$, and so there exists $f \in L^{1}(Z)$ such that $\left\|f_{n}-f\right\|_{L^{1}} \rightarrow 0$. Then $P f_{n}(B) \rightarrow P f(B)$ for each $B \in V$, and so $d\left(P f_{n}\right)(e) \rightarrow d(P f)(e)$ for each $e \in E$. Since $d\left(P f_{n}\right)$ is uniformly bounded in $\ell^{p, \infty}(E)$, this implies that $d(P f) \in$ $\ell^{p, \infty}(E)$ by Lemma 2.3 , and so $f \in A^{p}(Z)$. Moreover, using the same lemma, we also see that

$$
\left\|d\left(P f_{n}\right)-d(P f)\right\|_{\ell p, \infty} \rightarrow 0,
$$

and so $f_{n} \rightarrow f$ in $A^{p}(Z)$ for $n \rightarrow \infty$ as desired.

Given $u: V \rightarrow \mathbb{R}$ we define a type of maximal function $\mathcal{M} u: V \rightarrow$ $[0, \infty]$ by setting

$$
(\mathcal{M} u)(B)=\sum_{B^{\prime} \in \bigcup_{k=n}^{\infty} V_{k}, 8 B^{\prime} \cap 8 B \neq \emptyset} \frac{\left|B^{\prime}\right|}{|B|}\left|u\left(B^{\prime}\right)\right|
$$

for $B \in V_{n}, n \in \mathbb{N}_{0}$. There is nothing special about the constant 8 in the condition $8 B^{\prime} \cap 8 B \neq \emptyset$. Its usefulness will become apparent later in the proof of Corollary 4.6.

Lemma 4.3. Let $p>1$. Then there exists a constant $C \geq 0$ such that

$$
\|\mathcal{M} u\|_{\ell^{p, \infty}} \leq C\|u\|_{\ell^{p, \infty}}
$$

for each $u \in \ell^{p, \infty}(V)$.

In other words, the sublinear operator $u \mapsto \mathcal{M} u$ is bounded in $\ell^{p, \infty}(V)$.

For $Z=\mathbb{R}^{n}$ this lemma is essentially $\underline{\mathrm{ABH}}$, Lemma 4.6, p. 711]; see also [RS, p. 269].

Proof. First note that the operator $\mathcal{M}$ can be obtained from a kernel $k: V \times V \rightarrow[0, \infty)$. Indeed, we define

$$
\mathcal{K}\left(B, B^{\prime}\right)=\left|B^{\prime}\right| /|B|
$$

if $B \in V_{n}, B^{\prime} \in V_{k}, k, n \in \mathbb{N}, k \geq n$, and $8 B \cap 8 B^{\prime} \neq \emptyset$, and $\mathcal{K}\left(B, B^{\prime}\right)=$ 0 otherwise. Then for $u: V \rightarrow \mathbb{R}$ and $B \in V$ we have

$$
\mathcal{M} u(B)=\sum_{B^{\prime} \in V} \mathcal{K}\left(B, B^{\prime}\right)\left|u\left(B^{\prime}\right)\right| .
$$


The main point is that this kernel $\mathcal{K}$ satisfies a Schur condition (see [Wo, pp. 86-87]): if $q>1, \alpha \in(0,1 / q)$ and $g(B)=|B|^{\alpha}$ for $B \in V$, then

$$
\begin{aligned}
\sum_{B^{\prime} \in V} \mathcal{K}\left(B, B^{\prime}\right) g\left(B^{\prime}\right)^{q /(q-1)} & =\frac{1}{|B|} \sum_{k=n}^{\infty} \sum_{B^{\prime} \in V_{k}, 8 B \cap 8 B^{\prime} \neq \emptyset}\left|B^{\prime}\right|^{1+\alpha q /(q-1)} \\
& \lesssim \sum_{k=n}^{\infty} 2^{-k Q \alpha q /(q-1)} \\
& \simeq 2^{-n Q \alpha q /(q-1)} \simeq g(B)^{q /(q-1)}
\end{aligned}
$$

for $B \in V_{n}, n \in \mathbb{N}_{0}$, and

$$
\begin{aligned}
\sum_{B \in V} g(B)^{q} \mathcal{K}\left(B, B^{\prime}\right) & =\left|B^{\prime}\right| \sum_{n=0}^{k} \sum_{B \in V_{n}, 8 B \cap 8 B^{\prime} \neq \emptyset}|B|^{-1+\alpha q} \\
& \simeq\left|B^{\prime}\right| \sum_{n=0}^{k} 2^{-n Q(-1+\alpha q)} \simeq\left|B^{\prime}\right| 2^{-k Q(-1+\alpha q)} \\
& \simeq 2^{-k Q \alpha q} \simeq\left|B^{\prime}\right|^{\alpha q} \simeq g\left(B^{\prime}\right)^{q}
\end{aligned}
$$

for $B^{\prime} \in V_{k}, k \in \mathbb{N}_{0}$. This implies that the operator $\mathcal{M}$ is bounded in $\ell^{q}(V)$ for each $q>1$ and hence bounded in $\ell^{p, \infty}(V)$ by interpolation [SW, Chapter V.3].

Lemma 4.4. Let $p>1$.

(i) If $u, u^{\prime}: V \rightarrow \mathbb{R}$ with $d u \in \ell^{p, \infty}(E)$ and $u-u^{\prime} \in \ell^{p, \infty}(V)$, then $d u^{\prime} \in \ell^{p, \infty}(E)$ and $\mathbb{R} u=\mathbb{R} u^{\prime}$.

(ii) If $u: V \rightarrow \mathbb{R}$ and $d u \in \ell^{p, \infty}(E)$, then

$$
\begin{aligned}
\|u-P(\mathbb{R} u)\|_{\ell^{p, \infty}} & \lesssim\|d u\|_{\ell^{p, \infty}} \quad \text { and } \\
\| d\left(P(\mathbb{R} u) \|_{\ell^{p, \infty}}\right. & \lesssim\|d u\|_{\ell^{p, \infty}} .
\end{aligned}
$$

In particular, $u-P(\mathbb{R} u) \in \ell^{p, \infty}(V)$.

Proof. (i) We have

$$
\begin{aligned}
\left\|d u^{\prime}\right\|_{\ell^{p, \infty}} & \leq\left\|d\left(u^{\prime}-u\right)\right\|_{\ell^{p, \infty}}+\|d u\|_{\ell^{p, \infty}} \\
& \lesssim\left\|u^{\prime}-u\right\|_{\ell^{p, \infty}}+\|d u\|_{\ell^{p, \infty}}<\infty
\end{aligned}
$$

and so both $\mathbb{R} u$ and $\mathbb{R} u^{\prime}$ are defined by Lemma 4.1.

If $w: V \rightarrow \mathbb{R}$ is a function with $w \in \ell^{p, \infty}(V)$, then $|w(B)|$ is small if the level of $B \in V$ is large enough. Hence $\sup _{x \in Z}\left|\left(T_{n} w\right)(x)\right| \rightarrow 0$ and so $\left\|T_{n} w\right\|_{L^{1}} \rightarrow 0$ as $n \rightarrow \infty$. If we apply this to $w=u-u^{\prime}$, the claim follows. 
(ii) If $u$ is as in the statement, then an estimate as in (12) implies that for $B \in V_{n}$ with $n \in \mathbb{N}_{0}$ we have,

$$
\begin{aligned}
& |u(B)-P(\mathbb{R} u)(B)| \leq \\
& \left|u(B)-\frac{1}{|B|} \int_{B} T_{n} u d \mu\right|+\sum_{k=n}^{\infty} \frac{1}{|B|} \int_{B}\left|T_{k} u-T_{k+1} u\right| d \mu \\
& \leq \sum_{B^{\prime} \in \cup_{k=n}^{\infty} V_{k}, B^{\prime} \cap B \neq \emptyset} \frac{\left|B^{\prime}\right|}{|B|} \tilde{d} u\left(B^{\prime}\right) \leq \mathcal{M}(\tilde{d} u)(B) .
\end{aligned}
$$

Here $\mathcal{M}(\tilde{d} u)$ is as defined in (14). The first inequality now follows from Lemma 4.3 and (9).

For the second inequality note that

$$
\begin{aligned}
\|d(P(\mathbb{R} u))\|_{\ell^{\infty}, p} & \lesssim\|d(u-P(\mathbb{R} u))\|_{\ell^{\infty}, p}+\|d u\|_{\ell^{\infty}, p} \\
& \lesssim\|u-P(\mathbb{R} u)\|_{\ell^{\infty}, p}+\|d u\|_{\ell^{\infty}, p} \\
& \lesssim\|d u\|_{\ell^{\infty}, p} .
\end{aligned}
$$

Proof of Theorem 1.3. If $[u] \in \mathrm{Wk}^{1, p}(X)$, then $d u \in \ell^{p, \infty}(E)$, and so $\mathbb{R} u \in L^{1}(Z)$ is defined by Lemma 4.1. Moreover, by Lemma 4.4 (ii) we have

$$
\|d(P(\mathbb{R} u))\|_{\ell^{\infty, p}} \lesssim\|d u\|_{\ell^{\infty, p}}<\infty,
$$

and so $\mathbb{R} u \in A^{p}(Z)$. Note that $\mathbb{R} u$ is well-defined in $A^{p}(Z)$ and depends only on $[u]$; indeed, if $u^{\prime} \in[u]$, then there exists a constant $c \in \mathbb{R}$ such that $u-u^{\prime}-c \in \ell^{p, \infty}(V)$. Then by Lemma 4.4 (i) we have $\mathbb{R} u=\mathbb{R}\left(u^{\prime}+c\right)=c+\mathbb{R} u^{\prime}$. So we see that $[u] \in \mathrm{Wk}^{1, Q}(X) \mapsto \mathbb{R} u \in$ $A^{p}(Z)$ defines a linear map $\mathbb{R}: \mathrm{Wk}^{1, p}(X) \rightarrow A^{p}(Z)$.

It is clear that $[u] \in \mathrm{Wk}^{1, p}(X) \mapsto\|u\|_{\mathrm{Wk}^{1, p}}$ defines a semi-norm on $\mathrm{Wk}^{1, p}(X)$. Suppose that $\|u\|_{\mathrm{Wk}^{1, p}}=0$ for $[u] \in \mathrm{Wk}^{1, p}(X)$. By taking the infimum in (15) over all representatives in $[u]$ we see that

$$
\|\mathbb{R} u\|_{A^{p}(Z)}=\|d(P(\mathbb{R} u))\|_{\ell^{\infty, p}}=0,
$$

and so $\mathbb{R} u$ is constant; but then $u \in \ell^{p, \infty}(V)+\mathbb{R}$ by Lemma 4.4 (ii), showing that $[u]=0$. Hence $[u] \mapsto\|u\|_{\mathrm{Wk}^{1, p}}$ is a norm on $\mathrm{Wk}^{1, p}(X)$. Inequality (15) also shows that $\mathbb{R}: \mathrm{Wk}^{1, p}(X) \rightarrow A^{p}(Z)$ is a bounded linear operator from the normed space $\mathrm{Wk}^{1, p}(X)$ into the Banach space $A^{p}(Z)$.

It is immediate from Lemma 4.2 that the map $f \in A^{p}(Z) \mapsto[P f] \in$ $\mathrm{Wk}^{1, p}(X)$ is a bounded linear operator $\mathcal{P}: A^{p}(Z) \rightarrow \mathrm{Wk}^{1, p}(X)$ such that the composition $\mathbb{R} \circ \mathcal{P}$ is the identity on $A^{p}(Z)$. If $[u] \in \mathrm{Wk}^{1, p}(X)$, then, by what we have seen, $d(P(\mathbb{R} u)) \in \ell^{p, \infty}(E)$. Moreover, $P(\mathbb{R} u)-$ $u \in \ell^{p, \infty}(V)$ by Lemma 4.4 (ii). Hence $[u]=[P(\mathbb{R} u)]$ which shows that 
$\mathcal{P} \circ \mathbb{R}$ is the identity on $\mathrm{Wk}^{1, p}(X)$. It follows that $\mathbb{R}$ is an isomorphism between the normed spaces $\mathrm{Wk}^{1, p}(X)$ and $A^{p}(Z)$ with the inverse given by the operator $\mathcal{P}$. Since $A^{p}(Z)$ is a Banach space, $\mathrm{Wk}^{1, p}(X)$ is a Banach space as well. The proof is complete.

In order to show that $A^{p}(Z)$ does not depend on the filling $X$ in any essential way, we need a lemma.

Lemma 4.5. Let $p>1$, and $f \in L^{1}(Z)$ with $d(P f) \in \ell^{p, \infty}(E)$. Define $D f: V \rightarrow \mathbb{R}$ as

$$
(D f)(B)=\frac{1}{|8 B|} \int_{8 B}\left|f-f_{B}\right| d \mu
$$

for $B \in V$. Then

$$
\|D f\|_{\ell^{p, \infty}} \leq C\|d(P f)\|_{\ell^{p, \infty}},
$$

where $C \geq 0$ is constant independent of $f$.

Proof. Let $u=P f$. Then by Lemma 4.1 and Lemma 4.2 we have $f=\mathbb{R}(P f)=\lim _{n \rightarrow \infty} T_{n} u$ with convergence in $L^{1}(Z)$ and pointwise almost everywhere on $Z$. It follows that for $B \in V_{n}, n \in \mathbb{N}_{0}$, we have

$$
\begin{aligned}
(D f)(B) & =\frac{1}{|8 B|} \int_{8 B}\left|f-f_{B}\right| d \mu \\
& \leq \frac{1}{|8 B|} \int_{8 B}\left|u(B)-T_{n} u\right| d \mu+\frac{1}{|8 B|} \sum_{k=n}^{\infty} \int_{8 B}\left|T_{k+1} u-T_{k} u\right| d \mu \\
& \lesssim \frac{1}{|B|} \sum_{B^{\prime} \in \bigcup_{k=n}^{\infty} V_{k}, B^{\prime} \cap 8 B \neq \emptyset}\left|B^{\prime}\right| \tilde{d} u\left(B^{\prime}\right) \leq \mathcal{M}(\tilde{d} u)(B),
\end{aligned}
$$

where $\mathcal{M}$ is defined as in (14). By Lemma 4.3 and (9) this implies

$$
\|D f\|_{\ell^{p, \infty}} \lesssim\|\mathcal{M}(\tilde{d} u)\|_{\ell^{p, \infty}} \lesssim\|\tilde{d} u\|_{\ell^{p, \infty}} \lesssim\|d u\|_{\ell^{p, \infty}}
$$

and the claim follows.

Corollary 4.6. For $p>1$ is space is $\dot{A}^{p}(Z)$ is independent of the hyperbolic filling with comparability of semi-norms for different fillings.

Proof. Suppose $X^{\prime}=\left(V^{\prime}, E^{\prime}\right)$ is a hyperbolic filling of $Z$ constructed as in the beginning of this section using possibly different maximal $2^{-n}$ separated sets $Z_{n}^{\prime}$. Then again $V^{\prime}$ is a collection of balls, and we denote by $P^{\prime} f: V^{\prime} \rightarrow \mathbb{R}$ the Poisson extension of a function $f \in L^{1}(Z)$, and by $d u^{\prime}: E^{\prime} \rightarrow \mathbb{R}$ the gradient of a function $u^{\prime}: V^{\prime} \rightarrow \mathbb{R}$. It suffices to show that $\left\|d\left(P^{\prime} f\right)\right\|_{\ell^{p, \infty}} \simeq\|d(P f)\|_{\ell^{p, \infty}}$ for $f \in L^{1}(Z)$. For this it is enough to show that $\left\|d\left(P^{\prime} f\right)\right\|_{\ell^{p, \infty}} \lesssim\|d(P f)\|_{\ell^{p, \infty}}$ by symmetry. 
So suppose $f \in L^{1}(Z)$, and define $u^{\prime}=P^{\prime} f$. Let $e^{\prime} \in E^{\prime}$ be arbitrary, and $n \in \mathbb{N}_{0}$ be the level of $Q:=e_{+}^{\prime}$. Then there exists $B \in V_{n}$ such that $B \cap Q \neq \emptyset$. Since the radii of $B$ and $Q$ agree, and the radius of $Q^{\prime}=e_{-}^{\prime}$ is at most twice as large as the radius of $Q$ we have $Q, Q^{\prime} \subset 8 B$ (this inclusion is the reason why we choose the constant 8 in the definition (14) of $\mathcal{M})$. It follows that

$$
\begin{aligned}
\left|d\left(P^{\prime} f\right)\left(e^{\prime}\right)\right| & =\left|f_{Q}-f_{Q^{\prime}}\right| \leq\left|f_{Q}-f_{B}\right|+\left|f_{Q^{\prime}}-f_{B}\right| \\
& \leq \frac{1}{|Q|} \int_{Q}\left|f-f_{B}\right| d \mu+\frac{1}{\left|Q^{\prime}\right|} \int_{Q^{\prime}}\left|f-f_{B}\right| d \mu \\
& \lesssim \frac{1}{|8 B|} \int_{8 B}\left|f-f_{B}\right| d \mu=(D f)(B) .
\end{aligned}
$$

Here we used that $|Q| \simeq\left|Q^{\prime}\right| \simeq|8 B|$. Note that for a given ball $B \in V$ there is only a uniformly bounded number of edges $e^{\prime} \in E^{\prime}$ so that $B$ can appear in (16). Hence Lemma 2.2 and Lemma 4.5 imply that

$$
\left\|d\left(P^{\prime} f\right)\right\|_{\ell^{p, \infty}} \lesssim\|D f\|_{\ell^{p, \infty}} \lesssim\|d(P f)\|_{\ell^{p, \infty}}
$$

as desired.

Remark 4.7. In the previous proof we insisted on all hyperbolic fillings to be constructed from the scale range $2^{-n}$. There is no difficulty in extending the scope of Corollary 4.6 to hyperbolic fillings obtained from an arbitrary scale range $\Lambda^{-n}$ with $\Lambda>1$. This only requires some adjustment of the constant 8 in the definition (14) of the maximal operator $\mathcal{M}$. This leads to more general versions of Lemma 4.5 that can be used to extend Corollary 4.6. We omit the details, because this only leads to technicalities adding nothing of substance.

\section{SOBOLEV SPACES}

In this section $Z=(Z, d, \mu)$ is again an Ahlfors $Q$-regular compact metric measure space with $Q>0$, and $X=(V, E)$ the hyperbolic filling of $Z$.

If $\alpha>0$ and $f \in L^{1}(Z)$, then we denote by $\mathcal{D}_{\alpha}(f)$ the set of all measurable functions $g \geq 0$ on $Z$ for which there exists a set $N \subset Z$ with $|N|=0$ such that

$$
|f(x)-f(y)| \leq d(x, y)^{\alpha}(g(x)+g(y))
$$

for all $x, y \in Z \backslash N$.

For $\alpha>0$ and $p \geq 1$ we denote by $\dot{M}^{\alpha, p}(Z)$ the (homogeneous) fractional Hajtasz-Sobolev space consisting of all integrable functions $f \in L^{1}(Z)$ such that there exists a function $g \in \mathcal{D}_{\alpha}(f)$ with $g \in L^{p}(Z)$; in other words, $f \in \dot{M}^{\alpha, p}(Z)$ if there exists a non-negative function 
$g \in L^{p}(Z)$ such that (17) is true for almost every $x$ and $y$ in $Z$. The spaces $\dot{M}^{\alpha, p}(Z)$ were introduced in $[\mathrm{Ha}$. for $\alpha=1$ and in $\mathrm{Ya}$. for arbitrary $\alpha>0$. We define a semi-norm on $\dot{M}^{\alpha, p}(Z)$ by setting

$$
\|f\|_{\dot{M}^{\alpha, p}}=\inf \left\{\|g\|_{L^{p}}: g \in \mathcal{D}_{\alpha}(f)\right\} .
$$

Lemma 5.1. Let $p>1, \alpha>0$, and $f \in L^{1}(Z)$. Suppose $\left\{f_{n}\right\}$ is a sequence in $L^{1}(Z)$ such that $f_{n}(x) \rightarrow f(x)$ as $n \rightarrow \infty$ for almost every $x \in Z$. Then

$$
\|f\|_{\dot{M}^{\alpha, p}} \leq \liminf _{n \rightarrow \infty}\left\|f_{n}\right\|_{\dot{M}^{\alpha, p}}
$$

In particular, if the sequence $\left\{f_{n}\right\}$ lies in $\dot{M}^{\alpha, p}(Z)$ and $\left\|f_{n}\right\|_{\dot{M}^{\alpha, p}}$ is uniformly bounded for $n \in \mathbb{N}$, then $f \in \dot{M}^{\alpha, p}(Z)$.

Proof. If $C_{0}:=\liminf _{n \rightarrow \infty}\left\|f_{n}\right\|_{\dot{M}^{\alpha, p}}=\infty$ there is nothing to prove. So let us assume that $C_{0}<\infty$ and pick $C>C_{0}$. Then by passing to a subsequence if necessary, we may assume that $\left\|f_{n}\right\|_{\dot{M}^{\alpha, p}}<C$ for all $n \in \mathbb{N}$. Hence for each $n \in \mathbb{N}$ there exists $g_{n} \in \mathcal{D}_{\alpha}\left(f_{n}\right)$ such that $\left\|g_{n}\right\|_{L^{p}}<C$. So the sequence $\left\{g_{n}\right\}$ is uniformly bounded in $L^{p}(Z)$. Again by passing to a subsequence, we may assume that $g_{n} \rightarrow g \in L^{p}(Z)$ with respect to the weak-* topology in $L^{p}(Z)$. By Mazur's lemma [Yo, Section V.1] we can find a sequence $\left\{\tilde{g}_{n}\right\}$ in $L^{p}(Z)$, where each function $\tilde{g}_{n}$ is a finite convex combination of functions $g_{k}$ with $k \geq n$, such that we have norm-convergence $\tilde{g}_{n} \rightarrow g$ in $L^{p}(Z)$. Then $\left\|\tilde{g}_{n}\right\|_{L^{p}} \leq C$ for all $n \in \mathbb{N}$ and hence also $\|g\|_{L^{p}} \leq C$.

Note that condition (17) passes to convex combinations of pairs $(f, g)$. So by using the same coefficients as in the definition of $\tilde{g}_{n}$, we can find a convex combination $\tilde{f}_{n}$ involving the functions $f_{k}$ with $k \geq n$ such that $\tilde{g}_{n} \in \mathcal{D}_{\alpha}\left(\tilde{f}_{n}\right)$. Then we still have $\tilde{f}_{n}(x) \rightarrow f(x)$ as $n \rightarrow \infty$ for almost every $x \in Z$. By passing to yet another subsequence, we may assume that $\tilde{g}_{n}(x) \rightarrow g(x)$ for almost every $x \in Z$. Then by using (17) for the pair $\left(\tilde{f}_{n}, \tilde{g}_{n}\right)$ and passing to the pointwise limit in $x$ and $y$ outside a suitable set $N \subset Z$ with $|N|=0$, we see that $g \in \mathcal{D}_{\alpha}(f)$. So $\|f\|_{\dot{M}^{\alpha, p}} \leq C$. The claim follows by letting $C \rightarrow C_{0}$.

Lemma 5.2. Let $p \geq 1, \alpha>0$, and $f \in L^{1}(Z)$. If $\|f\|_{\dot{M}^{\alpha, p}}=0$, then $f$ is constant almost everywhere on $Z$.

Proof. If $\|f\|_{\dot{M}^{\alpha, p}}=0$, then there exists a sequence $\left\{g_{n}\right\}$ of functions in $\mathcal{D}_{\alpha}(f)$ with $\left\|g_{n}\right\|_{L^{p}} \rightarrow 0$ as $n \rightarrow \infty$. By passing to a suitable subsequence, we may assume that $g_{n}(x) \rightarrow 0$ as $n \rightarrow \infty$ for almost every $x \in Z$. A limiting argument then implies that the constant function $g_{\infty} \equiv 0$ lies in $\mathcal{D}_{\alpha}(f)$. Hence $f$ is constant almost everywhere on $Z$. 
If $g \in L^{1}(Z)$, then we we define the Hardy-Littlewood maximal function by

$$
(M g)(x)=\sup _{x \in B} \frac{1}{|B|} \int_{B}|g| d \mu,
$$

for $x \in Z$, where the supremum is taken over all balls $B \subset Z$ with $x \in B$. It is a standard fact that for $p>1$ the operator $f \mapsto M(f)$ is bounded in $L^{p}(Z)$ [He, pp. 10-12].

Proposition 5.3. Let $p>1$, and $\alpha=Q / p$. Then $\dot{M}^{\alpha, p}(Z) \subset \dot{A}^{p}(Z)$. Moreover, there exists a constant $C \geq 0$ such that

$$
\|f\|_{\dot{A}^{p}(Z)} \leq C\|f\|_{\dot{M}^{\alpha, p}}
$$

for all $f \in \dot{M}^{\alpha, p}(Z)$.

Proof. Let $p>1, \alpha=Q / p$, and $f \in \dot{M}^{\alpha, p}(Z)$. Then there exists $g \in \mathcal{D}_{\alpha}(f)$ with $g \in L^{p}(Z)$. If $u=P f$, then we have to show that $d u \in \ell^{p, \infty}(E)$ with a suitable norm bound.

For $\lambda>0$ let us denote by $E(\lambda)$ the collection of all edges $e \in E$ such that $|d u(e)|>\lambda$ and by $V(\lambda)$ the collection of all corresponding balls $B=e_{+}$, where $e \in E(\lambda)$. Then we have

$$
\# E(\lambda) \lesssim \# B(\lambda) \leq \int_{Z}\left(\sum_{B \in V(\lambda)} \frac{1}{|B|} \chi_{B}\right) d \mu,
$$

where $\chi_{B}$ denotes the characteristic function of $B$. Moreover, by the dyadic structure of the balls $B$ we have

$$
\sum_{B \in V(\lambda)} \frac{1}{|B|} \chi_{B}(z) \lesssim \frac{1}{\left|B_{z}\right|},
$$

where $B_{z}$ is a ball in $V_{\lambda}$ of smallest radius that contains $z \in Z$ if such a ball exists.

We consider $e \in E$, and let $B=e_{+}$and $B^{\prime}=e_{-}$. Note that $\operatorname{diam}(B) \simeq|B|^{1 / Q}$ and $\operatorname{diam}(B)^{\alpha} \simeq|B|^{1 / p}$. Then for a sufficiently large constant $\Lambda \geq 1$ independent of $e$ we have

$$
\begin{aligned}
|d u(e)| & =\left|u(B)-u\left(B^{\prime}\right)\right|=\left|f_{B}-f_{B^{\prime}}\right| \\
& \leq \frac{1}{|B| \cdot\left|B^{\prime}\right|} \int_{B} \int_{B^{\prime}}|f(x)-f(y)| d \mu(x) d \mu(y) \\
& \lesssim \frac{\operatorname{diam}(B)^{\alpha}}{|B| \cdot\left|B^{\prime}\right|} \int_{B} \int_{B^{\prime}}(g(x)+g(y)) d \mu(x) d \mu(y) \\
& \lesssim|B|^{1 / p} \frac{1}{|\Lambda B|} \int_{\Lambda B} g d \mu \lesssim|B|^{1 / p}(M g)(z),
\end{aligned}
$$


whenever $z \in B$. Here $M g$ is the Hardy-Littlewood maximal function of $g$ as in (18). The previous inequality shows that

$$
|d u(e)|>\lambda \Rightarrow \frac{1}{|B|} \lesssim \frac{1}{\lambda^{p}}(M(g)(z))^{p} \text { for all } z \in B=e_{+} .
$$

Note that this implies that if $z \in Z$ and there exist balls $B \in V(\lambda)$ of arbitrarily small radius with $z \in B$, then $M(g)(z)=+\infty$. Otherwise, we can apply (20). This leads to the inequality

$$
\sum_{B \in V(\lambda)} \frac{1}{|B|} \chi_{B} \lesssim \frac{1}{\lambda^{p}} M(g)^{p}
$$

Substituting this into (19) and integrating over $Z$, we finally obtain

$$
\# E(\lambda) \lesssim \frac{1}{\lambda^{p}} \int_{X} M(g)^{p} d \mu \lesssim \frac{1}{\lambda^{p}} \int_{X} g^{p} d \mu .
$$

Here we used $p>1$ and that the maximal function is bounded in $L^{p}(Z)$. Inequality (22) implies

$$
\|f\|_{\dot{A}^{p}}=\|d(P f)\|_{\ell^{p, \infty}} \lesssim\|g\|_{L^{p}}<\infty,
$$

and so $f \in A^{p}(Z)$. Taking the infimum over all $g \in \mathcal{D}_{\alpha}(f)$ in the last inequality, we conclude that $\|f\|_{A^{p}} \lesssim\|f\|_{\dot{M}^{\alpha, p}}$ as desired.

For a Lipschitz function $f: Z \rightarrow \mathbb{R}$ and $x \in Z$ we define

$$
\operatorname{Lip}_{x} f=\limsup _{r \rightarrow 0^{+}} \sup _{y \in B(x, r)}|f(y)-f(x)| / r,
$$

and a measurable function $\operatorname{Lip} f: Z \rightarrow \mathbb{R}$ by setting $(\operatorname{Lip} f)(x)=$ $\operatorname{Lip}_{x} f$ for $x \in Z$. For $p \geq 1$ we say that $Z$ supports a $p$-Poincaré inequality if there exist constants $C \geq 0$ and $\Lambda \geq 1$ such that

$$
\frac{1}{|B|} \int\left|f-f_{B}\right| d \mu \leq C R\left(\frac{1}{|\Lambda B|} \int_{\Lambda B}(\operatorname{Lip} f)^{p} d \mu\right)^{1 / p}
$$

for every Lipschitz function $f: Z \rightarrow \mathbb{R}$ and every ball $B \subset Z$, where $R$ is the radius of $B$. Note that our definition is equivalent to the more standard one given in [He]; see [Ke, Theorem 2].

The following lemma is essentially well-known.

Lemma 5.4. Let $(Z, d, \mu)$ be an Ahlfors $Q$-regular compact metric measure space that supports a $Q$-Poincaré inequality, where $Q>1$. Then there exists a constant $C>0$ such that

$$
\|f\|_{\dot{M}^{1, Q}} \leq C\|\operatorname{Lip} f\|_{L^{Q}}
$$

for every Lipschitz function $f: Z \rightarrow \mathbb{R}$. 
Proof. By [KZ] we know that there exists $r \in[1, Q)$ such that $(Z, d, \mu)$ supports an $r$-Poincaré inequality.

Let $f: Z \rightarrow \mathbb{R}$ be a Lipschitz function and $x, y \in Z$ with $x \neq y$ be arbitrary. Define $\rho=\operatorname{Lip} f$ and $B_{n}=B\left(x, 2^{-n} d(x, y)\right)$ for $n \in \mathbb{N}_{0}$. Then the $r$-Poincaré inequality implies that for some constant $\Lambda \geq 1$ we have

$$
\begin{aligned}
\left|f(x)-f_{B_{0}}\right| & \leq \sum_{n=0}^{\infty}\left|f_{B_{n}}-f_{B_{n+1}}\right| \\
& \lesssim d(x, y) \sum_{n=0}^{\infty} 2^{-n}\left(\frac{1}{\left|\Lambda B_{n}\right|} \int_{\Lambda B_{n}}(\operatorname{Lip} f)^{r} d \mu\right)^{1 / r} \\
& \lesssim d(x, y)\left[M\left(\rho^{r}\right)(x)\right]^{1 / r} .
\end{aligned}
$$

Here $M$ denotes the Hardy-Littlewood maximal function defined as in (18).

If we define $B_{0}^{\prime}=B(y, d(x, y))$, then very similar estimates lead to

$$
\left|f(y)-f_{B_{0}^{\prime}}\right| \lesssim d(x, y)\left[M\left(\rho^{r}\right)(y)\right]^{1 / r}
$$

and

$$
\left|f_{B_{0}}-f_{B_{0}^{\prime}}\right| \lesssim d(x, y)\left[M\left(\rho^{r}\right)(x)\right]^{1 / r} .
$$

Hence

$$
|f(x)-f(y)| \lesssim d(x, y)\left(\left[M\left(\rho^{r}\right)(x)\right]^{1 / r}+\left[M\left(\rho^{r}\right)(y)\right]^{1 / r}\right),
$$

where the implicit multiplicative constant is independent of $f, x, y$. It follows that for some constant $k_{0}$ independent of $f$ we have $k_{0}\left[M\left(\rho^{r}\right)\right]^{1 / r} \in$ $\mathcal{D}_{1}(f)$. Hence

$$
\begin{aligned}
\|f\|_{\dot{M}^{1, Q}}^{Q} & \lesssim\left\|\left[M\left(\rho^{r}\right)\right]^{1 / r}\right\|_{L^{Q}}^{Q}=\int_{Z}\left[M\left(\rho^{r}\right)\right]^{Q / r} d \mu \\
& \lesssim \int_{Z}\left[\rho^{r}\right]^{Q / r} d \mu=\int_{Z}(\operatorname{Lip} f)^{Q} d \mu=\|\operatorname{Lip} f\|_{L^{Q}}^{Q} .
\end{aligned}
$$

Here we used that $Q / r>1$ which implies that $g \mapsto M(g)$ is bounded in $L^{Q / r}(Z)$. The claim follows.

The previous argument actually shows that if $p \leq Q$ is sufficiently close to $Q$ (namely if $p \in(r, Q]$ ), then $\|f\|_{\dot{M}^{1, p}} \lesssim\|\operatorname{Lip} f\|_{L^{p}}$.

Proof of Theorem 1.4. Let $Z$ be as in the statement, and $X=(V, E)$ be the hyperbolic filling of $Z$ as discussed in Section 4. By Proposition 5.3 we know that $\dot{M}^{1, Q}(Z) \subset \dot{A}^{Q}(Z)$ with a suitable semi-norm bound.

For the other direction, let us assume that $f \in L^{1}(Z)$ and $u=P f$ satisfies $d u \in \ell^{Q, \infty}(E)$. Fix $N \in \mathbb{N}$ and consider the gradient functions 
$\tilde{d}_{n} u$ on levels $n \in[N, 2 N]$. The total number of vertices corresponding to these levels is $\simeq 2^{2 N Q}$. Hence

$$
\sum_{n=N}^{2 N}\left\|\tilde{d}_{n} u\right\|_{\ell^{Q}}^{Q} \lesssim N\|d u\|_{\ell, \infty}^{Q}
$$

as follows from (9) and the first inequality in Lemma 2.1 (ii). In particular, there exists $n \in[N, 2 N]$ such that

$$
\left\|\tilde{d}_{n} u\right\|_{\ell Q} \lesssim\|d u\|_{\ell Q, \infty}
$$

By using this fact on a non-overlapping sequence of such intervals $[N, 2 N]$ one can find a sequence $\left\{n_{k}\right\}$ in $\mathbb{N}$ with $n_{k} \rightarrow \infty$ and

$$
\left\|\tilde{d}_{n_{k}} u\right\|_{\ell Q} \lesssim\|d u\|_{\ell Q, \infty}
$$

for all $k \in \mathbb{N}$. Then $\left\|\tilde{d}_{n_{k}} u\right\|_{\ell^{Q}}$ stays uniformly bounded as $k \rightarrow \infty$.

By Lemma 4.1 and Lemma 4.2 we have

$$
f=\mathbb{R}(P f)=\mathbb{R} u=\lim _{k \rightarrow \infty} T_{n_{k}} u
$$

where the limit is in $L^{1}(Z)$ and pointwise almost everywhere in $Z$. So $f \in \dot{M}^{1, Q}(Z)$ will follow from Lemma 5.1, if we can show that the norms $\left\|T_{n_{k}} u\right\|_{\dot{M}^{1, Q}}$ are uniformly bounded for $k \in \mathbb{N}$.

To see this, let $n \in \mathbb{N}$ and $x \in Z$. We pick $B \in V_{n}$ with $x \in B$. Then for $y \in Z$ we have,

$$
\left|T_{n} u(y)-T_{n} u(x)\right| \leq \sum_{B^{\prime} \in V_{n}}\left|u\left(B^{\prime}\right)-u(B)\right| \cdot\left|\psi_{B^{\prime}}(y)-\psi_{B^{\prime}}(x)\right|,
$$

and so

$$
\begin{aligned}
\operatorname{Lip}_{x}\left(T_{n} u\right) & =\limsup _{r \rightarrow 0^{+}} \sup \left\{\left|T_{n} u(y)-T_{n} u(x)\right| / r: y \in Z, d(y, x)<r\right\} \\
& \leq \sum_{B^{\prime} \in V_{n}, x \in B^{\prime}}\left|u\left(B^{\prime}\right)-u(B)\right| \operatorname{Lip}_{x}\left(\psi_{B^{\prime}}\right) \\
& \lesssim 2^{n} \sum_{B^{\prime} \in V_{n}, x \in B^{\prime}}\left|u\left(B^{\prime}\right)-u(B)\right| \lesssim 2^{n} \tilde{d} u(B) .
\end{aligned}
$$

Here we used (10) and (11). Hence by Lemma 5.4 we have

$$
\left\|T_{n} u\right\|_{\dot{M}^{1, Q}}^{Q} \lesssim\left\|\operatorname{Lip}\left(T_{n} u\right)\right\|_{L^{Q}}^{Q} \lesssim \sum_{B \in V_{n}} \tilde{d} u(B)^{Q}=\left\|\tilde{d}_{n} u\right\|_{\ell Q}^{Q} .
$$

Applying this inequality on the subsequence $\left\{n_{k}\right\}$, we see that

$$
\begin{aligned}
\|f\|_{\dot{M}^{1, Q}} & \leq \liminf _{k \rightarrow \infty}\left\|T_{n_{k}} u\right\|_{\dot{M}^{1, Q}} \lesssim \liminf _{k \rightarrow \infty}\left\|\operatorname{Lip}\left(T_{n_{k}} u\right)\right\|_{L^{Q}} \\
& \lesssim \liminf _{k \rightarrow \infty}\left\|\tilde{d}_{n_{k}} u\right\|_{\ell^{Q}} \lesssim\|d u\|_{\ell^{Q, \infty}}<\infty
\end{aligned}
$$


So $f \in \dot{M}^{1, Q}(Z)$, and we also conclude that $\|f\|_{\dot{M}^{1, Q}} \lesssim\|f\|_{\dot{A}^{Q}}$. The proof is complete.

Proposition 5.5. Under the assumptions of Theorem 1.4, the space $\dot{A}^{p}(Z)$ consists only of constant functions for $1<p<Q$.

Proof. Let $1<p<Q, f \in A^{p}(Z)$, and $u=P f$. We pick $q \in(p, Q)$ close to $Q$. As in the proof of Theorem 1.4, one shows that there exists a sequence $\left\{n_{k}\right\}$ in $\mathbb{N}$ with $n_{k} \rightarrow \infty$ as $k \rightarrow \infty$ such that $\left\|\tilde{d}_{n_{k}} u\right\|_{\ell^{p}} \lesssim 1$. Since $q>p$, we then also have $\left\|\tilde{d}_{n_{k}} u\right\|_{\ell^{q}} \leq\left\|\tilde{d}_{n_{k}} u\right\|_{\ell^{p}} \lesssim 1$. Inequality (23) implies that

$$
\left\|\operatorname{Lip}\left(T_{n} u\right)\right\|_{L^{q}}^{q} \lesssim 2^{-(Q-q) n}\left\|\tilde{d}_{n} u\right\|_{\ell^{q}}^{q}
$$

and so $\left\|\operatorname{Lip}\left(T_{n_{k}} u\right)\right\|_{L^{q}} \rightarrow 0$ as $k \rightarrow \infty$. If $q$ is sufficiently close to $Q$, then we have (see the remark after the proof of Lemma 5.4),

$$
\begin{aligned}
\|f\|_{\dot{M}^{1, q}} & \leq \liminf _{n \rightarrow \infty}\left\|T_{n} u\right\|_{\dot{M}^{1, q}} \lesssim \liminf _{n \rightarrow \infty}\left\|\operatorname{Lip}\left(T_{n} u\right)\right\|_{L^{q}} \\
& \leq \lim _{k \rightarrow \infty}\left\|\operatorname{Lip}\left(T_{n_{k}} u\right)\right\|_{L^{q}}=0 .
\end{aligned}
$$

Hence $\|f\|_{\dot{M}^{1, q}}=0$, and so $f$ is a constant function by Lemma 5.2 .

\section{The EuClidean Setting}

We will now discuss results in the spirit of Theorem 1.4 in the standard Euclidean setting. We choose the simplest framework, where the underlying space is $Z=\mathbb{R}^{n}, n \in \mathbb{N}$, equipped with Lebesgue measure.

We denote Lebesgue measure of a measurable set $M \subset \mathbb{R}^{n}$ by $|M|$, and use $d x$ to indicate Lebesgue measure in integrals. We consider the hyperbolic filling $X$ of $Z=\mathbb{R}^{n}$ as given by upper-halfspace $\mathbb{R}_{+}^{n+1}=$ $\left\{(x, t): x \in \mathbb{R}^{n}, t>0\right\}$. In contrast to our earlier discussion, in this section we consider the (classical) Poisson extension $u=P f$ of a function $f \in L^{p}\left(\mathbb{R}^{n}\right)$, where $p \in[1, \infty]$. If

$$
P_{t}(x)=c_{n} \frac{t}{\left(t^{2}+|x|^{2}\right)^{(n+1) / 2}}, \quad x \in \mathbb{R}^{n}, t>0,
$$

is the Poisson kernel, where $c_{n}=\Gamma((n+1) / 2) / \pi^{(n+1) / 2}$, then $u$ is given by the convolution $u(x, t)=\left(P_{t} * f\right)(x)$ for $(x, t) \in \mathbb{R}_{+}^{n+1}$ (see St, Chapter 3.2] for general background).

If $\mu$ is a measure on set $A \subset \mathbb{R}^{n}$ and $p \geq 1$, then we define the weak-type space $L^{p, \infty}(A, \mu)$ as the space of all measurable functions $f: A \rightarrow \mathbb{R}$ for which there exists a constant $C \geq 0$ such that

$$
\mu(\{x \in A:|f(x)|>\lambda\}) \leq C^{p} / \lambda^{p}
$$

for all $\lambda>0$. We denote by $\|f\|_{L^{p, \infty}(A, \mu)}$ the infimum of all constants $C \geq 0$ for which this inequality is valid. 
We can now formulate a result, whose basic idea is found CST, Appendix]. It provides a somewhat surprising concrete embedding of $L^{p}$-spaces into subspaces of the weak-type spaces $L^{p, \infty}$.

Proposition 6.1. Let $p>1$ and $s>0$. If $f \in L^{p}\left(\mathbb{R}^{n}\right)$, then the Poisson extension $u=P f$ satisfies

$$
t^{s / p} u(x, t) \in L^{p, \infty}\left(\mathbb{R}_{+}^{n+1}, t^{-(s+1)} d x d t\right) .
$$

Conversely, if a harmonic function $u$ on $\mathbb{R}_{+}^{n+1}$ satisfies (24), then $u=$ Pf, where $f \in L^{p}\left(\mathbb{R}^{n}\right)$.

Of course, here $t^{s / p} u(x, t)$ stands for the function on $\mathbb{R}_{+}^{n+1}$ given by

$$
(x, t) \mapsto t^{s / p} u(x, t) .
$$

We will also make a similar abuse of notation below.

Proof. We denote by $\mu_{s}$ the measure on $\mathbb{R}_{+}^{n+1}$ given by

$$
d \mu_{s}(x, t)=t^{-(s+1)} d x d t .
$$

Let $f \in L^{p}\left(\mathbb{R}^{n}\right)$. Then $M f \in L^{p}\left(\mathbb{R}^{n}\right)$ for the Hardy-Littlewood maximal function of $f$, and a standard estimate for the Poisson extension [St, p. 62] gives the bound

$$
|u(x, t)| \leq c(M f)(x)
$$

for each $(x, t) \in \mathbb{R}_{+}^{n+1}$, where $c>0$ is a constant independent of $f$ and $x$.

Fix $x \in \mathbb{R}^{n}$ and $\lambda>0$. If for $t>0$ we have $\left|t^{s / p} u(x, t)\right|>\lambda$, then it follows that

$$
t \geq\left(\frac{\lambda}{c(M f)(x)}\right)^{p / s}
$$

We obtain

$$
\begin{aligned}
\mu_{s}\left\{(x, t) \in \mathbb{R}_{+}^{n+1}: \mid t^{s / p}\right. & u(x, t) \mid>\lambda\} \\
& \leq \int_{\mathbb{R}^{n}}\left(\int_{(\lambda / c(M f)(x))^{p / s}}^{\infty} \frac{d t}{t^{s+1}}\right) d x \\
& \leq \frac{c^{p}}{s \lambda^{p}} \int_{\mathbb{R}^{n}}[(M f)(x)]^{p} d x \lesssim \frac{1}{\lambda^{p}}\|f\|_{L^{p}}^{p},
\end{aligned}
$$

which implies the first direction of the statement.

In order to prove the other direction, let us assume that $u$ is harmonic in $\mathbb{R}_{+}^{n+1}$ and satisfies (24). We first want to show a pointwise estimate for $u$. So let $\left(x_{0}, t_{0}\right) \in \mathbb{R}_{+}^{n+1}$ be arbitrary, $R=t_{0} / 2$, and 
$B=B\left(\left(x_{0}, t_{0}\right), R\right)$ be the Euclidean ball of radius $R$ centered at $\left(x_{0}, t_{0}\right)$. Note that for points $(x, t) \in B$ we have $t \simeq R$ and so

$$
d \mu_{s}(x, t) \simeq R^{-(s+1)} d x d t
$$

on $B$. Hence (24) implies that

$$
|\{(x, t) \in B:|u(x, t)|>\lambda\}| \lesssim \frac{R}{\lambda^{p}}
$$

for each $\lambda>0$. Since $u$ is harmonic, it follows that

$$
\begin{aligned}
\left|u\left(x_{0}, t_{0}\right)\right| & \leq \frac{1}{|B|} \int_{B}|u(x, t)| d x d t \\
& \leq \frac{1}{|B|}\left(R^{-n / p}|B|+\int_{R^{-n / p}}^{\infty}|\{(x, t) \in B:|u(x, t)|>\lambda\}| d \lambda\right) \\
& \lesssim R^{-n / p} \simeq\left|t_{0}\right|^{-n / p} .
\end{aligned}
$$

This estimate implies that for each $\delta>0$ the function $u$ is bounded on $H_{\delta}:=\left\{(x, t) \in \mathbb{R}_{+}^{n+1}: t \geq \delta\right\}$. If we set $u_{\delta}=u(\cdot, \delta)$, then the integral representation for bounded harmonic functions [St, p. 199, Proposition 1] shows that

$$
u(x, t+\delta)=\left(P u_{\delta}\right)(x, t)
$$

for $(x, t) \in \mathbb{R}_{+}^{n+1}$. To obtain a representation for $u$ itself, we would like to pass to a limit here along a sequence $\delta_{k} \rightarrow 0^{+}$.

For this we fix $r \in(1, p)$, and write $u=b+v$, where

$$
b=\min \{\max \{u,-1\}, 1\}
$$

and $v=u-b$. Note that $|b| \leq 1$. We want to show that for suitable $\delta>0$ we have good $L^{r}$-norm bounds for $v(\cdot, \delta)$.

For this we consider the slab

$$
A_{k}:=\left\{(x, t) \in \mathbb{R}_{+}^{n+1}: 2^{-(k+1)}<t<2^{-k}\right\} .
$$

for $k \in \mathbb{N}$. Since

$$
d \mu_{s}(x, t) \simeq 2^{k(s+1)} d x d t
$$

on $A_{k}$, our assumption (24) implies that

$$
\left|\left\{(x, t) \in A_{k}:|u(x, t)|>\lambda\right\}\right| \lesssim \frac{2^{-k}}{\lambda^{p}}
$$

for $\lambda>0$. If $|v|>\lambda>0$, then $|u|>1+\lambda$, and so

$$
\begin{aligned}
\int_{A_{k}}|v(x, t)|^{r} d x d t & =r \int_{0}^{\infty} \lambda^{r-1}\left|\left\{(x, t) \in A_{k}:|v(x, t)|>\lambda\right\}\right| d \lambda \\
& \lesssim 2^{-k} \int_{0}^{\infty} \frac{\lambda^{r-1}}{(1+\lambda)^{p}} d \lambda \simeq 2^{-k} .
\end{aligned}
$$


Using Fubini's theorem we can find $\delta_{k}>0$ with $2^{-(k+1)}<\delta_{k}<2^{-k}$ such that for $v_{k}:=v\left(\cdot, \delta_{k}\right)$ we have $\left\|v_{k}\right\|_{L^{r}} \lesssim 1$. Let $b_{k}=b\left(\cdot, \delta_{k}\right)$. Then $\left|b_{k}\right| \leq 1$. So the sequences $\left\{v_{k}\right\}$ and $\left\{b_{k}\right\}$ are uniformly bounded in $L^{r}\left(\mathbb{R}^{n}\right)$ and $L^{\infty}\left(\mathbb{R}^{n}\right)$, respectively. By passing to a subsequence if necessary, we may assume that $v_{k} \rightarrow f_{1} \in L^{r}\left(\mathbb{R}^{n}\right)$ and $b_{k} \rightarrow f_{2} \in$ $L^{\infty}\left(\mathbb{R}^{n}\right)$ as $k \rightarrow \infty$, where convergence is with respect to the respective weak-* topologies. If $t>0$, then for the Poisson kernel $P_{t}$ we have $P_{t} \in L^{q}\left(\mathbb{R}^{n}\right)$ for all $q \in[1, \infty]\left[\right.$ St, p. 62]. It follows that $\left(P_{t} * v_{k}\right)(x) \rightarrow$ $\left(P_{t} * f_{1}\right)(x)$ and $\left(P_{t} * b_{k}\right)(x) \rightarrow\left(P_{t} * f_{1}\right)(x)$ as $k \rightarrow \infty$ for all $x \in \mathbb{R}^{n}$ and $t>0$. We now define $f=f_{1}+f_{2} \in L^{r}\left(\mathbb{R}^{n}\right)+L^{\infty}\left(\mathbb{R}^{n}\right)$. Note that $u_{\delta_{k}}=u\left(\cdot, \delta_{k}\right)=v_{k}+b_{k}$. Hence for each $(x, t) \in \mathbb{R}_{+}^{n+1}$ we have

$$
\begin{aligned}
u(x, t) & =\lim _{k \rightarrow \infty} u\left(x, t+\delta_{k}\right)=\lim _{k \rightarrow \infty}\left(P u_{\delta_{k}}\right)(x, t) \\
& =\lim _{k \rightarrow \infty}\left(P_{t} *\left(v_{k}+b_{k}\right)\right)(x)=\lim _{k \rightarrow \infty}\left(P_{t} * v_{k}\right)(x)+\lim _{k \rightarrow \infty}\left(P_{t} * b_{k}\right)(x) \\
& =\left(P_{t} * f_{2}\right)(x)+\left(P_{t} * f_{1}\right)(x)=(P f)(x, t) .
\end{aligned}
$$

In other words, $u=P f$ is the Poisson extension of $f$.

We want to show that $f \in L^{p}\left(\mathbb{R}^{n}\right)$. Since $f \in L^{r}\left(\mathbb{R}^{n}\right)+L^{\infty}\left(\mathbb{R}^{n}\right)$, where $r>1$, for almost every $x \in \mathbb{R}^{n}$ we have [St, p. 62, Theorem 1]

$$
f(x)=\lim _{t \rightarrow 0^{+}}(P f)(x, t)=\lim _{t \rightarrow 0^{+}} u(x, t) .
$$

In particular, since $u$ is continuous, we can find a measurable function $\varepsilon: \mathbb{R}^{n} \rightarrow[0, \infty]$ such that $\varepsilon(x)>0$ and

$$
|u(x, t)| \geq|f(x)| / 2 \quad \text { for } t \in(0, \varepsilon(x))
$$

for almost every $x \in \mathbb{R}^{n}$. Here we may assume that $\epsilon(x)=\infty$ if $f(x)=0$.

For $\lambda>0$ consider the set

$$
S_{\lambda}:=\left\{x \in \mathbb{R}^{n}: 2^{-p}|f(x)|^{p} \geq 2 \lambda^{p} \epsilon(x)^{-s}\right\}
$$

(with the understanding that $\epsilon(x)^{-s}=0$ if $\epsilon(x)=\infty$ ). Since $\epsilon(x)>0$ for almost every $x \in \mathbb{R}^{n}$, the set $S_{\lambda}$ increases to a full measure set as $\lambda \rightarrow 0^{+}$. If $x \in S_{\lambda}$, then $\left|t^{s / p} u(x, t)\right|>\lambda$ for $(2 \lambda /|f(x)|)^{p / s}<t<\varepsilon(x)$. We deduce that

$$
\begin{aligned}
\lambda^{p} \mu_{s}\left\{(x, t) \in \mathbb{R}_{+}^{n+1}\right. & \left.:\left|t^{s / p} u(x, t)\right|>\lambda\right\} \\
& \geq \lambda^{p} \int_{S_{\lambda}}\left(\int_{(2 \lambda /|f(x)|)^{p / s}}^{\epsilon(x)} t^{-(s+1)} d t\right) d x \\
& =\frac{1}{s} \int_{S_{\lambda}}\left(2^{-p}|f(x)|^{p}-\lambda^{p} \epsilon(x)^{-s}\right) d x
\end{aligned}
$$




$$
\geq \frac{2^{-p}}{2 s} \int_{S_{\lambda}}|f(x)|^{p} d x .
$$

By letting $\lambda \rightarrow 0^{+}$we conclude

$$
\|f\|_{L^{p}}^{p} \lesssim\left\|t^{s / p} u(x, t)\right\|_{L^{p, \infty}\left(\mathbb{R}_{+}^{n+1}, \mu_{s}\right)}^{p}<\infty .
$$

The claim follows.

In the next corollary we consider locally integrable functions $f$ on $\mathbb{R}^{n}$ that have distributional partial derivatives $\partial_{i} f$ in $L^{p}\left(\mathbb{R}^{n}\right)$ for $i=$ $1, \ldots, n$, where $p>1$. We denote by $\nabla f=\left(\partial_{1} f, \ldots, \partial_{n} f\right)$ the gradient of such a function $f$, and by $|\nabla f|$ the pointwise Euclidean norm of $\nabla f$.

Corollary 6.2. Let $p>1, s>0$, and $u=P f$, where $f \in L^{p}\left(\mathbb{R}^{n}\right)$. Then $|\nabla f| \in L^{p}\left(\mathbb{R}^{n}\right)$ if and only if

$$
t^{s / p}|\nabla u(x, t)| \in L^{p, \infty}\left(\mathbb{R}_{+}^{n+1}, t^{-(s+1)} d x d t\right) .
$$

Proof. We consider $u=P f$, where $f \in L^{p}\left(\mathbb{R}^{n}\right)$, and use the notation $u_{t}=u(\cdot, t)$ for $t>0$.

We first assume that $f$ has distributional partial derivatives $\partial_{i} f \in$ $L^{p}\left(\mathbb{R}^{n}\right)$ for $i=1, \ldots, n$. Then for each $t>0$ we have

$$
\partial_{i} u_{t}=\partial_{i}\left(P_{t} * f\right)=P_{t} * \partial_{i} f .
$$

This shows that all partial derivatives $\partial_{i} u$ of $u$ in the $x$-direction are Poisson extensions of functions in $L^{p}\left(\mathbb{R}^{n}\right)$. To get a similar statement also for the partial derivative $\partial_{t} u$ in the $t$-direction, we use the Riesz transforms $R_{i}, i=1, \ldots, n$ (see [St, p. 57] for the definition). Since $p>1$, these are bounded operators on $L^{p}\left(\mathbb{R}^{n}\right)$ [St, Chapter 3.1], and so we have $R_{i}\left(\partial_{i} f\right) \in L^{p}\left(\mathbb{R}^{n}\right)$. Moreover, if we define

$$
g=-\sum_{i=1}^{n} R_{i}\left(\partial_{i} f\right) \in L^{p}\left(\mathbb{R}^{n}\right),
$$

then $\partial_{t} u_{t}=P_{t} * g$. This can easily verified if $f$ is $C^{\infty}$-smooth and has compact support. The general case follows from the density of such functions in the Sobolev space $W^{1, p}\left(\mathbb{R}^{n}\right)$. We conclude that all partial derivatives of $u$ are Poisson extensions of functions in $L^{p}\left(\mathbb{R}^{n}\right)$. Condition (27) now follows from Proposition 6.1.

For the converse direction suppose that $u=P f$ satisfies (27). Then by Proposition 6.1 we know that for suitable functions $g_{1}, \ldots, g_{n} \in$ $L^{p}\left(\mathbb{R}^{n}\right)$ we have $\partial_{i} u_{t}=P_{t} * g_{i}$ for $i=1, \ldots, n$ and all $t>0$. Now standard properties of the Poisson kernel give the convergence $u_{t}=$ $P_{t} * f \rightarrow f$ and $\partial_{i} u_{t}=P_{t} * g_{i} \rightarrow g_{i}$ in $L^{p}\left(\mathbb{R}^{n}\right)$ as $t \rightarrow 0^{+}$[St, p. 62, Theorem 1]. This implies that $g_{i}$ is the distributional partial derivative 
$\partial_{i} f$ of $f$. So $\partial_{i} f=g_{i} \in L^{p}\left(\mathbb{R}^{n}\right)$ for $i=1, \ldots, n$ and the claim follows.

Remark 6.3. The previous corollary essentially gives a characterization of functions $f$ in the Sobolev space $W^{1, p}\left(\mathbb{R}^{n}\right)$ in terms of their Poisson extension $u$. For simplicity we made the a priori assumption $f \in L^{p}\left(\mathbb{R}^{n}\right)$. One can relax this integrability condition on $f$ and prove a more general result characterizing functions $f$ in the homogeneous Sobolev space $\dot{W}^{1, p}\left(\mathbb{R}^{n}\right)$ consisting of locally integrable function $f$ on $\mathbb{R}^{n}$ with distributional derivatives in $L^{p}\left(\mathbb{R}^{n}\right)$. For this one first checks that the Poisson extension is well-defined for each $f \in \dot{W}^{1, p}\left(\mathbb{R}^{n}\right)$. Then a generalization of the above corollary reads as follows: a locally integrable function $f$ on $\mathbb{R}^{n}$ belongs to the homogeneous Sobolev space $\dot{W}^{1, p}\left(\mathbb{R}^{n}\right)$ if and only if it satisfies the integrability condition $\int_{\mathbb{R}^{n}}|f(x)|(1+|x|)^{-(n+1)} d x<\infty$, and (27) is valid for its Poisson extension $u$.

The upper half-space $\mathbb{R}_{+}^{n+1}$ carries the usual hyperbolic metric. This is the Riemannian metric obtained from rescaling the Euclidean metric by the factor $1 / t$ at the point $(x, t)$. Corresponding to this metric, one has a hyperbolic gradient $\nabla_{h} u$ of a smooth function $u$ on $\mathbb{R}_{+}^{n+1}$ defined by $\nabla_{h} u(x, t)=t \nabla u(x, t)$ for $(x, t) \in \mathbb{R}_{+}^{n+1}$, and hyperbolic measure $\mu_{h}$ given by $d \mu_{h}=t^{-(n+1)} d x d t$. By specializing to the case $s=p=n \geq 2$ in Corollary 6.2, we obtain the following analog of Theorem 1.4.

Corollary 6.4. Let $n \geq 2$ and suppose $u=\operatorname{Pf}$, where $f \in L^{n}\left(\mathbb{R}^{n}\right)$. Then $|\nabla f| \in L^{n}\left(\mathbb{R}^{n}\right)$ if and only if $\left|\nabla_{h} u\right| \in L^{n, \infty}\left(\mathbb{R}_{+}^{n+1}, d \mu_{h}\right)$.

Most of the results in this section remain valid if the Poisson extension is replaced by other convolution approximations. We will not pursue this in detail, but limit ourselves to recording the following key fact whose proof we will only sketch.

Lemma 6.5. Let $p>1, f \in W^{1, p}\left(\mathbb{R}^{n}\right)$, and $k$ be a bounded and nonnegative kernel on $\mathbb{R}^{n}$ with

$$
0<\int_{\mathbb{R}^{n}}(1+|x|) k(x) d x<\infty .
$$

Define $u(x, t)=\int_{\mathbb{R}^{n}} f(x-y) k(y / t) t^{-n} d x$ for $(x, t) \in \mathbb{R}_{+}^{n+1}$. Then

$$
\|f\|_{W^{1, p}} \simeq\|f\|_{L^{p}}+\operatorname{ess} \sup _{t>0}\||\nabla u|(\cdot, t)\|_{L^{p}}
$$

Here $\nabla u$ has to be interpreted as the distributional gradient of $u$, and we take the essential supremum of the $L^{p}$-norm of $|\nabla u|(\cdot, t)$ for $t>0$. 
Note that condition (28) is not true for the Poisson kernel $k=P_{1}$.

Outline of proof. The statement follows from standard approximation properties of convolutions. The only issue that is not entirely straightforward is how to control the (distributional) $t$-derivative $\partial_{t} u$ in terms of the derivatives $\partial_{i} f$ of our given function $f \in W^{1, p}\left(\mathbb{R}^{n}\right)$.

For this we first assume in addition to our hypotheses that $k$ is smooth and compactly supported. Then computation shows that for $t>0$ and $y=\left(y_{1}, \ldots, y_{n}\right) \in \mathbb{R}^{n}$ we have

$$
\begin{aligned}
\frac{\partial}{\partial t}\left(t^{-n} k(y / t)\right) & =-n t^{-(n+1)} k(y / t)-t^{-(n+2)} \sum_{i=1}^{n} y_{i}\left(\partial_{i} k\right)(y / t) \\
& =-t^{-(n+1)} \sum_{i=1}^{n} \frac{\partial}{\partial y_{i}}\left(y_{i} k(y / t)\right) .
\end{aligned}
$$

Integration by parts then gives

$$
\partial_{t} u(x, t)=\sum_{i=1}^{n} \int_{\mathbb{R}^{n}} \partial_{i} f(x-y) v_{i}(y / t) t^{-n} d y,
$$

where $v_{i}(y):=y_{i} k(y)$. Our hypothesis (28) implies $k \in L^{1}\left(\mathbb{R}^{n}\right)$ and $v_{i} \in L^{1}\left(\mathbb{R}^{n}\right)$ for $i=1, \ldots, n$. An $L^{p}$-bound for $\partial_{t} u(\cdot, t)$ as desired immediately follows.

For general kernels $k$ one uses an approximation argument to reduce to the case of smooth and compactly supported kernels.

By using the previous lemma, one can (for example) replace the Poisson extension in Corollary 6.2 by the ball averages

$$
u(x, t)=\frac{1}{|B(x, t)|} \int_{B(x, t)} f(y) d y .
$$

In the proof one uses standard approximation properties of convolutions combined with the fact that the radial maximal function $\sup _{t>0} u(\cdot, t)$ is dominated by the Hardy-Littlewood maximal function. This last statement is true in greater generality if one demands, in addition to (28), that $k$ is a radially decaying function. For kernels that do not satisfy (28) such as the Poisson kernel, the issues become more involved.

\section{REFERENCES}

$[\mathrm{ABH}]$ K. Astala, M. Bonk, and J. Heinonen, Quasiconformal mappings with Sobolev boundary values, Ann. Sc. Norm. Super. Pisa Cl. Sci. (5) 1 (2002), 687-731.

[BP] M. Bourdon and H. Pajot, Cohomologie $l_{p}$ et espaces de Besov, J. Reine Angew. Math. 558 (2003), 85-108. 
[BS] S. Buyalo and V. Schroeder, Elements of asymptotic geometry, Europ. Math. Soc., Zürich, 2007.

[CST] A. Connes, D. Sullivan, and N. Teleman, Quasiconformal mappings, operators on Hilbert space, and local formulae for characteristic classes, Topology 33 (1994), 663-681.

[Gr] L. Grafakos, Classical Fourier analysis, 2nd ed., Springer, New York, 2008.

[Ha] P. Hajłasz, Sobolev spaces on an arbitrary metric space, Potential Anal. 5 (1996), 403-415.

[He] J. Heinonen, Lectures on analysis on metric spaces, Springer, New York, 2001.

[Ke] S. Keith, Modulus and the Poincaré inequality on metric measure spaces, Math. Z. 245 (2003), 255-292.

[KZ] S. Keith and X. Zhong, The Poincaré inequality is an open ended condition, Ann. of Math. (2) 167 (2008), 575-599.

[RS] R. Rochberg and S. Semmes, Nearly weakly orthonormal sequences, singular value estimates, and Calderón-Zygmund operators, J. Funct. Anal. 86 (1989), 237-306.

[Se] S. Semmes, Metric spaces and mappings seen at many scales, in: M. Gromov, Metric structures for Riemannian and non-Riemannian spaces, Birkhäuser, Boston, 1999, pp. 401-518.

[St] E. Stein, Singular integrals and differentiability properties of functions, Princeton Univ. Press, Princeton, N.J., 1970.

[SW] E. Stein and G. Weiss, Introduction to Fourier analysis on Euclidean spaces, Princeton Univ. Press, Princeton, N.J., 1971.

[Wo] P. Wojłaszczyk, Banach spaces for analysts, Cambridge Studies in Adv. Math. 25, Cambridge Univ. Press, Cambridge, 1991.

[Ya] D. Yang, New characterizations of Hajtasz-Sobolev spaces on metric spaces, Sci. China Ser. A 46 (2003), 675-689.

[Yo] K. Yosida, Functional analysis, Springer, Berlin-Heidelberg-New York, 1965.

Department of Mathematics, University of California, Los Angeles, Box 95155, Los Angeles, CA, 90095-1555, USA

E-mail address: mbonk@math.ucla.edu

Department of Mathematics and Statistics, University of Helsinki, PO Box 68, FI-00014 Helsinki, Finland

E-mail address: eero.saksman@helsinki.fi 